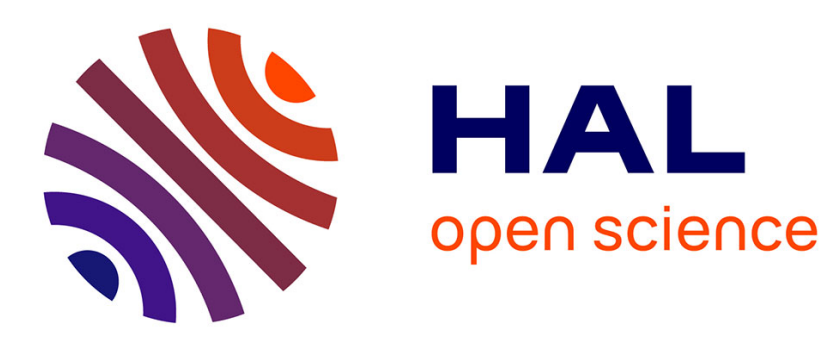

\title{
Lattice Dynamics Study of Elemental Bismuth under High Pressure
}

Yixuan Zhao, Sébastien Clément, Julien Haines, Romain Viennois

\section{To cite this version:}

Yixuan Zhao, Sébastien Clément, Julien Haines, Romain Viennois. Lattice Dynamics Study of Elemental Bismuth under High Pressure. Journal of Physical Chemistry C, 2020, 124 (49), pp.26659-26669. 10.1021/acs.jpcc.0c08371 . hal-03079788

\section{HAL Id: hal-03079788 \\ https://hal.science/hal-03079788}

Submitted on 17 Dec 2020

HAL is a multi-disciplinary open access archive for the deposit and dissemination of scientific research documents, whether they are published or not. The documents may come from teaching and research institutions in France or abroad, or from public or private research centers.
L'archive ouverte pluridisciplinaire HAL, est destinée au dépôt et à la diffusion de documents scientifiques de niveau recherche, publiés ou non, émanant des établissements d'enseignement et de recherche français ou étrangers, des laboratoires publics ou privés. 


\title{
Lattice dynamics study of elemental Bismuth under high pressure
}

Yixuan Zhao $^{1}$, Sébastien Clément ${ }^{2}$, Julien Haines ${ }^{1, *}$, Romain Viennois ${ }^{1, *}$

${ }^{1}$ ICGM, Université Montpellier, CNRS, ENSCM, Montpellier, France

${ }^{2}$ L2C, Université Montpellier, CNRS, ENSCM, Montpellier, France

\begin{abstract}
We report a combined experimental and theoretical study of the lattice dynamics of the different high pressure phases of the bismuth and their pressure dependence. The stability and the lattice dynamics of the different phases were calculated at room and high pressure. Raman scattering experiments were performed up to $9 \mathrm{GPa}$ and the Raman spectrum of the Bi-III phase with guest-host structure was measured for the first time. The assignment of the Raman modes of $\mathrm{Bi}-\mathrm{II}$ phase and the pressure dependence of the $\mathrm{Bi}-\mathrm{I}, \mathrm{Bi}-\mathrm{II}$ and $\mathrm{Bi}-\mathrm{III}$ phases were performed using DFT calculations. From the DFT calculations, we have determined the vibrational modes due to motion of the guest atoms along the $c$ direction.
\end{abstract}

* Corresponding authors: julien.haines@umontpellier.fr, romain.viennois@umontpellier.fr 


\section{Introduction}

Semimetallic $\mathrm{Bi}$ is one of the most investigated elements at high pressure due to its particular physico-chemical properties, interesting crystal structures under different pressures and complex phase diagram. It has wide applications such as a semi-metal, a low-dimensional thermoelectric material, a pressure calibrant and so on. ${ }^{1-3}$ Especially, its thermoelectric properties can be improved either by antimony substitution or by designing small diameter nanowires in which an energy bandgap opens, which improves its thermoelectric properties, especially at low temperatures. ${ }^{1,2}$

Under high pressure application, bulk bismuth has several different crystal structures, four of which can be stabilized at room temperature. They are shown in Figure $1 .{ }^{4-10}$ In the following, we will follow the notation proposed by Cannon in his review. ${ }^{3} \mathrm{Bi}-\mathrm{I}$ has a rhombohedral A7 type structure (R-3m space group) ${ }^{4}$, the monoclinic structure of phase II was determined by neutron diffraction ${ }^{11}$ to be $\mathrm{C}$-centered (C2/m space group) and its crystal structure was better refined very recently. ${ }^{5}$ The structure of $\mathrm{Bi}$-III has been described as monoclinic ${ }^{12}$, Tetragonal ${ }^{13}$ and orthorhombic ${ }^{14}$ in the 1970s. However, none of these structures fitted the experimental data, which were also not very accurate. Afterwards, in 1995, Chen et al ${ }^{15}$ has proposed a tetragonal structure, which is in good agreement with phase III XRD peak positions. In the most recent work, M. I. McMahon et al ${ }^{16}$ performed new more accurate diffraction experiments using synchrotron angle-dispersive powder diffraction and showed that the Chen's model had two main weaknesses: firstly, it cannot describe additional small Bragg reflexions and secondly, it cannot reproduce well the pressure dependence of the volume of elemental bismuth determined in prior works. ${ }^{16,17}$ They reported that $\mathrm{Bi}$-III has a more complex host-guest structure, which solves these contradictions. This structure has a tetragonal host and an interpenetrating tetragonal guest, which is incommensurate with the host along the host $\mathrm{c}$ axis. Later on, from synchrotron single-crystal X-ray diffraction, McMahon and coworkers have 
shown the presence of structural modulations in the Bi-III phase. ${ }^{18}$ The phase $\mathrm{V}$ is a body centered cubic (bcc) structure ${ }^{10}$ and stable up to $220 \mathrm{GPa} .{ }^{19}$ The overall increase in $\mathrm{Bi}$ coordination number with pressure is from 3 to 8 .

At room temperature, the Bi-I transforms to $\mathrm{Bi}-\mathrm{II}$ at $2.52 \mathrm{GPa} .{ }^{20}$ The Bi-II structure exhibits a narrow stability range up to $2.7 \mathrm{GPa} .{ }^{21}$ The I-II and II-III phase transitions are widely used for pressure calibration at $25^{\circ} \mathrm{C} .{ }^{3,22}$ The Bi-III is stable between $2.7 \mathrm{GPa}$ and $7.7 \mathrm{GPa} .{ }^{23}$ Finally, bcc $\mathrm{Bi}-\mathrm{V}$ is obtained from $\mathrm{Bi}-\mathrm{III}$ at higher pressure. There are still several uncertainties within the Bi-III stability field between 4 and $6.5 \mathrm{GPa}$ in which possible additional phase transition have been proposed. ${ }^{3,22,24-34}$ However, the existence of these transitions have not been convincingly confirmed until now and additional work is needed.

At high temperature and high pressure, an additional phase called Bi-IV was discovered first by Bundy ${ }^{21}$ and confirmed soon after by Klement. ${ }^{25}$ In the first structural study performed using neutron diffraction, Fedotov described this structure as tetragonal although not all Bragg peaks can be described with this proposed structure, ${ }^{14}$ whereas two decades later Chen et al performed a powder X-ray diffraction study using synchrotron radiation and described this structure as monoclinic. ${ }^{35}$ Degtyareva reinterpreted the data of both experiments and proposed to describe the Bi-IV structure as C-centered orthorhombic (Cmce space group) structure which is pseudo-tetragonal and very closely related to the monoclinic phase proposed earlier ${ }^{36}$ and this interpretation was confirmed only recently by a single-crystal X-ray diffraction study using synchrotron radiation by Chamayo et al. ${ }^{7}$ The existence of another HP-HT II' phase was proposed with very narrow existence range between about 1.7 and $2 \mathrm{GPa}$ and at about 180 to $192^{\circ} \mathrm{C}$ based on thermal analysis experiments. ${ }^{37}$ However, no other study confirmed the existence of such a phase before the recent work of Principi et al in $2006 .{ }^{6}$ Indeed, during the exploration of the metastable $\mathrm{P}-\mathrm{T}$ phase diagram of $\mathrm{Bi}$, they found the existence of a tetragonal phase they call $\beta$-Bi because it has the same crystal structure as $\beta-\mathrm{Sn} .{ }^{6}$ The boundaries of the 
stability range of this phase were between 1 and $2 \mathrm{GPa}$ and between 125 and $200^{\circ} \mathrm{C}$ and therefore was in the same region of the P-T phase diagram of Bi as II' phase, but with a much larger stability range. Thus, they propose that this $\beta$-Bi phase is the II' phase previously found by Tikhomirova et al. ${ }^{37}$ Very recently, Lin et al have confirmed the existence of this phase and the identification of $\beta$-Bi phase with the II' phase and also challenged the established melting lines of Bismuth above $1.5 \mathrm{GPa} .{ }^{38,39}$ The existence of the II' phase was also observed by two more recent works on schock-compressed Bi. ${ }^{40,41}$ A metastable phase with unkown crystal structure was observed during shock compression of $\mathrm{Bi} .{ }^{40,42}$ Among the different high pressure phases, the phases stable at low temperatures (phases II, III and V) are superconducting up to 9 $\mathrm{K}$ at $8 \mathrm{GPa} .{ }^{31,43-45}$

From the above review of the polymorphs of bismuth, one can see that there are still uncertainties about the stability of the different high pressure phases. Using DFT calculations, Haussermann et al ${ }^{9}$ have studied the stability and electronic structures of the I, II, III and V phases at $0 \mathrm{~K}$, but did not study the case of the HP-HT Bi-IV and $\beta$-Bi phases because their exact crystal structures were not known at that time and have been experimentally determined only recently. In recent papers on $\mathrm{Cu}-\mathrm{Bi}$ alloys under HP-HT, Amsler and coworkers 46,47 studied the stability of HP Bi phases (Bi-II, Bi-III, Bi-IV and Bi-V phases) by using DFT calculations. Gorman and coworkers studied the stability of HP Bi phases (Bi-II, $\beta$-Bi, Bi-III phases) by using DFT calculations. ${ }^{40}$ Thus, it seems interesting to study both the stability and electronic structures of the HP-HT $\beta$-Bi and Bi-IV phases compared to the other HP Bi phases.

Giving the exotic nature of some HP crystal structures of elemental Bi such as the host-guest structure Bi-III, a study of their lattice dynamics is interesting notably to understand the guest dynamics in the Bi-III phase as it can permit a better understanding of the coupling between phonons and electrons, which produces the low-T superconductivity in HP phases of Bi. Until now, the DFT lattice dynamics calculations using different exchange-correlation functionals 
have focused on the simpler phase $\mathrm{I}^{48-54}$ and only one such calculations was performed on phase V . ${ }^{55}$ Only very recently, two new studies have focused on calculations of the lattice dynamics of the Bi-III phase, but only using DFT. ${ }^{43,44}$ The phonon density of states has been calculated for the Bi-II phase very recently. ${ }^{45}$ From the experimental point of view, the study of different Bi phases by Raman spectroscopy have already been performed for the low-pressure phases BiI ${ }^{55-58}$ and Bi-II. ${ }^{58} \mathrm{H}$. Olijnyk et al ${ }^{58}$ measured for the first time the Raman spectrum of Bi-II at pressure up to $2.7 \mathrm{GPa}$, two peaks were observed around $40 \mathrm{~cm}^{-1}$ and $80 \mathrm{~cm}^{-1}$. Due to its metallic nature, it is difficult to measure the Raman spectrum of Bi-III. Therefore, in this work, high-pressure studies were extended up to $9.5 \mathrm{GPa}$ at room temperature in order to study the behavior of Bi-I and Bi-II and also attempt to measure Raman spectra for Bi-III and Bi-V.

\section{Experimental and computational details}

Bi powder of $99.5 \%$ purity was purchased from Alfa Aesar (E23Z002). Bi pieces of 99.999\% purity were purchased from Aldrich (7440-69-9) and ground into small grains. We performed two types of experiments using diamond anvil cell and rhenium gaskets. Bi powder was loaded into gasket hole with $150 \mu \mathrm{m}$ diameter and with indented part of $50 \mu \mathrm{m}$ thickness in the absence of a pressure-transmitting medium for the first experiment (non-hydrostatic). In the second experiment, the hole was filled with Bi grains and argon, the latter acted as a quasi-hydrostatic pressure-transmitting medium. The pressure was measured by ruby fluorescence method. ${ }^{59}$ Two experiments were performed using a Jobin-Yvon T64000 triple monochromator in combination with a liquid-nitrogen-cooled charge-coupled device detector. The $659.4 \mathrm{~nm}$ and $785.55 \mathrm{~nm}$ lines from diode lasers were used for all of the compression runs, the $514.5 \mathrm{~nm}$ line of an $\mathrm{Ar}^{+}$laser was used for some decompression runs in order to study possible resonant Raman effect, but without success. 
The first-principles calculations were performed within the Density Functional Theory (DFT) with the wave-plane VASP package using Projector Augmented Wave (PAW) pseudopotentials 60,61 within the standard Generalized Gradient Approximation (GGA) exchange-correlation functional of Perdew, Burke and Erzenhof (PBE) type. ${ }^{62}$ In all calculations, we used a Bi pseudopotential including the 5d electrons among the valence electrons and an energy cut-off of $350 \mathrm{eV}$. In order to reduce the computational cost, especially for the phonon calculations of the Bi-III phase, we have not included the spin-orbit interaction in our calculations as the effect is expected to be small in lattice dynamics. In all relaxation and phonon calculations, we used the first order Methfessel-Paxton method. ${ }^{63}$ The high density k-mesh samplings used were $15 \times 15 \times 15,15 \times 15 \times 15,21 \times 21 \times 21,11 \times 11 \times 7,9 \times 15 \times 15$ and $21 \times 21 \times 21$ for the Bi-I, Bi-II, $\beta$-Bi, BiIII (Haussermann's model ${ }^{9}$ ), Bi-IV and $\mathrm{Bi}-\mathrm{V}$, respectively and ensure that the relaxed structures are well converged. The different structures were relaxed until the residual forces were smaller than $10^{-4} \mathrm{eV} / \mathrm{A}$. Prior to the phonon calculations, the atomic positions were relaxed until the residual forces were smaller than $10^{-5} \mathrm{eV} / \AA$. We used the direct method for the phonon calculations. In the case of the Bi-I, Bi-II , $\beta$-Bi, Bi-IV and Bi-V, we used $4 \times 4 \times 4,2 \times 2 \times 4,2 \times 2 \times 4$, $1 \times 2 \times 2$ and $4 \times 4 \times 4$ supercells, respectively. In the case of the $\mathrm{Bi}-\mathrm{III}$, in order to reduce the computational cost and because the primitive cell size was large enough, the calculations were performed on a $1 \times 1 \times 1$ supercell, i. e. on the primitive cell, and the atoms were displaced by $0.03 \AA$ from their equilibrium positions in order to obtain accurate enough results. The Phonon code of K. Parlinski was used for the diagonalization of the dynamical matrix. ${ }^{64}$ The electronic structures of the different structures were also calculated using the same cut-off energies and kmesh sampling (with the exception of the Bi-II structure for which we used 25x25x25 k-mesh sampling), but the tetrahedron method was used for Brillouin zone integration. ${ }^{65}$ The calculations under pressure were performed by applying hydrostatic pressure and then relaxing 
the structure for each considered pressure. Thus, the phonon and electronic calculations were performed in a similar way as for the calculations at zero pressure.

\section{Results}

In the first step of our calculations, we studied the stability of the different known crystal structures of elemental bismuth in function of the pressure. Concerning the rhombohedral Bi-I, one finds the atomic volume $\mathrm{V}=36.225 \AA^{3}$ slightly larger than the experimental atomic volume $\mathrm{V}=34.985 \AA^{3},{ }^{66}$ which is usual with GGA exchange-correlation functional. The calculated atomic volumes and the formation enthalpies of the different elemental Bi phases at different pressures were calculated in order to evaluate the stability of the different structures.

Concerning the Bi-III phase, the work of McMahon et al has resolved the problem by finding a complex composite incommensurate structure. ${ }^{16} \mathrm{We}$ have calculated at different pressures the enthalpy $\mathrm{H}$ and the volume of the structure proposed by McMahon with the commensurate approximation made by Haussermann et al. ${ }^{9}$ In this case, the ratio of the lattice parameters c of the host structure and of the guest structure is: $c_{H} / c_{G}=4 / 3=1.3333 .{ }^{9}$ This is close to the experimental value $\mathrm{c}_{\mathrm{H}} / \mathrm{c}_{\mathrm{G}}=1.309(1) .{ }^{16}$ Recently, Chen et al ${ }^{34}$ found that the Haussermann's commensurate structure is much more stable than the first structures previously proposed for the Bi-III by Kabalkina et al ${ }^{12}$ and by Dugger et al ${ }^{13}$ and later discarded following the more accurate experiments of McMahon et al. ${ }^{16}$ More recently, Kartoon and Makov have calculated the $\mathrm{Bi}-\mathrm{III}$ structure for different ratio $\mathrm{c}_{\mathrm{H}} / \mathrm{c}_{\mathrm{G}}$ and found the modulation as in the experiments for large supercell. ${ }^{67}$ From this work, $\mathrm{c}_{\mathrm{H}} / \mathrm{c}_{\mathrm{G}}=4 / 3$ seems a reasonable approximation. In Figure 2, the pressure dependence of the calculated normalized volume $\mathrm{V} / \mathrm{V}_{0}$ and enthalpy difference $\Delta \mathrm{H}$ of the different structure is shown. The volume of the different phases is normalized with respect to the room pressure volume of the $\mathrm{Bi}-\mathrm{I}$ phase $\mathrm{V}_{0}$ and the enthalpy difference $\Delta \mathrm{H}$ is equal to $\mathrm{H}-\mathrm{H}(\mathrm{Bi}-\mathrm{I})$ where $\mathrm{H}$ is the enthalpy of a given $\mathrm{Bi}$ phase and $\mathrm{H}(\mathrm{Bi}-\mathrm{I})$ is the enthalpy 
of the Bi-I phase at the same pressure. For comparison, we also give the pressure dependence of the normalized volume obtained from different experimental set-ups. ${ }^{5,16}$

From our calculations, one can see the following sequence of stability at $0 \mathrm{~K}$ :

$$
\mathrm{I} \stackrel{3.12 \mathrm{GPa}}{\Longrightarrow} \beta-\mathrm{Bi} \stackrel{3.37 \mathrm{GPa}}{=} \mathrm{III} \stackrel{10.1 \mathrm{GPa}}{\Longrightarrow} \mathrm{IV} \stackrel{14.5 \mathrm{GPa}}{=} \mathrm{V}
$$

We find that the $\beta$-Bi phase is the most stable structure among the HP structures of elemental $\mathrm{Bi}$ at $0 \mathrm{~K}$. This contrasts with the work of Gorman et al who found that Bi-II is more stable than the $\beta$-Bi (or Bi-II') phase between 3 and $4.4 \mathrm{GPa} .{ }^{40}$ When we did not consider the $\beta$-Bi phase, one can see that the II phase would never be stable because its formation enthalpy is always higher than that of the phase III, which would be stabilized at $3.23 \mathrm{GPa}$, in rather good agreement with Haussermann et al who find this phase stable at about $3.54 \mathrm{GPa} .{ }^{9}$ Thus, if we consider only the case of the I, II, III and V phases, both the pressure dependence of their formation enthalpy and of their volume agree well between our work and that of Haussermann et al. ${ }^{9}$ In contrast to our work and of that of Haussermann et al, ${ }^{9}$ Amsler and coworkers found that the II phase can be stabilized with respect to phases I and III at $0 \mathrm{~K}$ between 2 and $2.8 \mathrm{GPa}$. ${ }^{46,47}$ Note that as in our work, they found that phase IV can be stabilized at $0 \mathrm{~K}$, but at slightly lower pressure (about 7.5GPa) and that phase $\mathrm{V}$ becomes the most stable phase at about 12-13 GPa. ${ }^{46,47}$ Kartoon and Makov also found that Bi-II is the most stable phase between 1.9 and 3.6 GPa, but they did not perform calculations neither for $\beta$-Bi nor Bi-IV. ${ }^{67}$ As DFT calculations are performed at $0 \mathrm{~K}$, we must compare with the P-T phase diagram of elemental $\mathrm{Bi}$ at the lowest temperatures. When decreasing the temperature below room temperature, the stability domain of the $\mathrm{Bi}$-II phase becomes narrower and some studies suggest even that this phase becomes unstable at the lowest temperature, permitting thus a direct transition from $\mathrm{Bi}-\mathrm{I}$ to $\mathrm{Bi}-\mathrm{III}$ phases as in the DFT calculations. ${ }^{3,22}$ In this case, the transition pressure $\mathrm{P}_{1}$ between $\mathrm{Bi}-\mathrm{I}$ and $\mathrm{Bi}-\mathrm{III}$ can be extrapolated at $0 \mathrm{~K}$ and this would be slightly above $3 \mathrm{GPa},{ }^{3,22}$ which is quite close to our results. However, Khasanov studied recently the superconducting properties 
of Bi-II at very low temperatures. ${ }^{45}$ There are still some controversies concerning the low-T phase diagram of elemental $\mathrm{Bi}$. But the above discussion shows that the $\mathrm{Bi}-\mathrm{II}$ phase is stabilized when the temperature increases, which means that some additional temperature dependent contribution such as vibrational or electronic entropies can stabilize it against the Bi-I and BiIII phases. However, we found that the $\beta$ - $\mathrm{Bi}$ is the most stable phase between 3.12 and 3.37 GPa. From our lattice dynamics calculations (see below), one can calculate the Gibbs energy at 2.5 GPa of the Bi-I, Bi-II, $\beta$-Bi and Bi-III phases. In Figure 3, one can see that indeed, the $\beta$-Bi and $\mathrm{Bi}-\mathrm{II}$ phases become more stable than the $\mathrm{Bi}-\mathrm{I}$ phase when increasing the temperature. The temperature at which the $\beta$-Bi becomes the most stable phase is however underestimated compared to the experiments. This means that the stability of the $\beta$-Bi phase is overestimated compared to other phases. Since Haussermann et al's work, ${ }^{9}$ the crystal structure of Bi-IV phase, which is stable at both high temperature and high pressure, has been solved by Chaimayo et $\mathrm{al}^{7}$ and we have carried out stability calculations of this phase to compare with the other elemental Bi phases. We find that this Bi-IV phase becomes slightly more stable than the BiIII above $10.1 \mathrm{GPa}$. This contrasts with the experimental data showing that the Bi-IV phase can be stabilized only at high temperatures (above $175^{\circ} \mathrm{C}$ ) and at lower pressure (between 2.1 and $5.4 \mathrm{GPa}){ }^{3,22} \mathrm{We}$ have calculated the Gibbs energies of the Bi-III and Bi-IV phases at $5 \mathrm{GPa}$ (see Fig. 3) and found that the Bi-IV phase becomes the most stable phase when increasing the temperature as in the experiments, but at lower temperature. This means that the stability of the $\mathrm{Bi}-\mathrm{IV}$ phase is overestimated compared to other phases. In our calculations, the $\mathrm{Bi}-\mathrm{V}$ phase is stable at much higher pressure (about $14.5 \mathrm{GPa}$ ) than in experiments (about $7.5 \mathrm{GPa}$ at $300 \mathrm{~K}$ and maybe $9 \mathrm{GPa}$ when extrapolating to $0 \mathrm{~K}$ the curve of the phase transformation $\mathrm{Bi}-\mathrm{III} / \mathrm{Bi}$ V). Similar results were found by Haussermann et al ${ }^{9}$ and by Amsler and coworkers. ${ }^{46,47}$ When comparing our calculated pressure variation of the volume with the experimental data (see Figure 2), the agreement is rather satisfactory although the transition pressures are not 
always correct, especially given that the DFT calculations were performed at $0 \mathrm{~K}$, whereas the experiments were carried out at room temperature.

Our results concerning the nature and stability of the $\beta$-Bi phase together with the recent experimental observations of this phase ${ }^{38-41}$ suggests a new interpretation of the thermal analysis experiments of Yoon et al: ${ }^{68}$ the metastable phase observed could be the $\beta$-Bi phase instead of the Bi-II phase.

As Haussermann et al ${ }^{9}$ have already reported the electronic structure of the Bi-I, Bi-II, Bi-III and Bi-IV and we found similar results. In Figure 4, we report the electronic density of states of $\beta$-Bi and $\mathrm{Bi}-\mathrm{II}$ at $2.5 \mathrm{GPa}$ and of $\mathrm{Bi}-\mathrm{IV}$ and $\mathrm{Bi}-\mathrm{III}$ at $5 \mathrm{GPa}$. All phases are metallic. The electronic density of states of $\mathrm{Bi}-\mathrm{II}$ and $\beta$-Bi have very similar shapes with slightly larger density of states $\mathrm{g}\left(\mathrm{E}_{\mathrm{F}}\right)$ for the $\beta$-Bi phase $(0.525$ states/eV.at $)$ than for the Bi-II phase $(0.455$ states/eV.at). The shape of the electronic density of states of the Bi-III and Bi-IV are different with a slightly larger density of states $g\left(E_{F}\right)$ for the Bi-IV phase $(0.535$ states/eV.at) than for the Bi-III phase (0.43 states/eV.at).

We have studied the lattice dynamics of the different Bi polymorphs using DFT calculations as well as of the different phases stable at room temperature using Raman spectroscopy experiments.

For the different polymorphs, the vibrational selection rules strongly change depending of the crystal structure and of the number of atoms. For the rhombohedral I structure with 2 atoms/unit cell, we have the following decomposition of the vibrational irreducible representations: $\Gamma=\mathrm{A}_{1 \mathrm{~g}}$ $\oplus \mathrm{E}_{\mathrm{g}} \oplus \mathrm{A}_{2 \mathrm{u}}(\mathrm{z}) \oplus \mathrm{E}_{\mathrm{u}}(\mathrm{x}, \mathrm{y})$. In this case, the modes with $\mathrm{A}_{2 \mathrm{u}}$ and $\mathrm{E}_{\mathrm{u}}$ symmetry are the acoustic modes and there are two Raman-active modes of $\mathrm{A}_{1 \mathrm{~g}}$ and $\mathrm{E}_{\mathrm{g}}$ symmetry and no infrared-active modes.

For the C-centered monoclinic II structure with 2 atoms/unit cell, we have the following decomposition of the vibrational irreducible representations: $\Gamma=2 \mathrm{~A}_{\mathrm{g}} \oplus \mathrm{B}_{\mathrm{g}} \oplus \mathrm{A}_{\mathrm{u}}(\mathrm{y}) \oplus 2 \mathrm{~B}_{\mathrm{u}}$ 
$(\mathrm{x}, \mathrm{z})$. In this case, the modes with $\mathrm{A}_{\mathrm{u}}$ and $\mathrm{B}_{\mathrm{u}}$ symmetries are the acoustic modes and there are three Raman-active modes of $\mathrm{A}_{\mathrm{g}}$ and $\mathrm{B}_{\mathrm{g}}$ symmetries and no infrared-active modes.

The decomposition of the vibrational irreducible representations of the $\beta$-Bi with 2 atoms/unit cell is: $\Gamma=\mathrm{A}_{1 \mathrm{~g}} \oplus \mathrm{E}_{\mathrm{g}} \oplus \mathrm{A}_{2 \mathrm{u}}(\mathrm{z}) \oplus \mathrm{E}_{\mathrm{u}}(\mathrm{x}, \mathrm{y})$. In this case, the modes with $\mathrm{A}_{2 \mathrm{u}}$ and $\mathrm{E}_{\mathrm{u}}$ are the acoustic modes and there are Raman-active modes of $\mathrm{A}_{1 \mathrm{~g}}$ and $\mathrm{E}_{\mathrm{g}}$ symmetries and no infrared-active modes.

So for studying the lattice dynamics of these phases, Raman spectroscopy is the spectroscopic tool of choice.

For the composite tetragonal III structure with 32 atoms/unit cell, when using the commensurate approximation in the space group ( $\mathrm{P} 4 / \mathrm{ncc}$ ), we have the following decomposition of the vibrational irreducible representations:

$\Gamma=6 \mathrm{~A}_{1 \mathrm{~g}} \oplus 7 \mathrm{~A}_{2 \mathrm{~g}} \oplus 5 \mathrm{~B}_{1 \mathrm{~g}} \oplus 4 \mathrm{~B}_{2 \mathrm{~g}} \oplus 13 \mathrm{E}_{\mathrm{g}} \oplus 6 \mathrm{~A}_{1 \mathrm{u}} \oplus 7 \mathrm{~A}_{2 \mathrm{u}} \oplus 5 \mathrm{~B}_{1 \mathrm{u}} \oplus 4 \mathrm{~B}_{2 \mathrm{u}} \oplus 13 \mathrm{E}_{\mathrm{u}}$. In this case, the acoustic modes have $\mathrm{A}_{2 \mathrm{u}}$ and $\mathrm{E}_{\mathrm{u}}$ symmetry and there are 28 Raman-active modes of $A_{1 g}, B_{1 g}, B_{2 g}$ and $E_{g}$ symmetries and 18 infrared-active modes of $A_{2 u}$ and $E_{u}$ symmetries. We must note that the selection rules for the guest atoms give 6 Raman active modes $\left(2 \mathrm{~A}_{1 \mathrm{~g}} \oplus 4\right.$ $\left.\mathrm{E}_{\mathrm{g}}\right)$.

As discussed by Wang et al. ${ }^{69}$ about high pressure Sb-II phase, that is isostructural with Bi-III, the host lattice can be described in a first approximation as the $\mathrm{Al}$ sublattice in $\mathrm{CuAl}_{2}$, i. e. by the $8 \mathrm{~h}$ site in the $\mathrm{I} 4 / \mathrm{mcm}$ structure. In this case, there are four Raman-active modes of $\mathrm{A}_{1 \mathrm{~g}}, \mathrm{~B}_{1 \mathrm{~g}}$, $\mathrm{B}_{2 \mathrm{~g}}$ and $\mathrm{E}_{\mathrm{g}}$ symmetries. In this simplified model, Degtyareva et al. ${ }^{70}$ described the host lattice by the (2a) site in the I4/mmm structure and in this case there are no Raman-active mode due to the guest atoms. With this simplified model, one expects 4 Raman-active modes and this model appears too simple and not enough realistic for well describing the complex crystal structure of Bi-III phase and the details of its Raman spectrum. Indeed, later on McMahon et al. ${ }^{18}$ have shown that there is a structural modulation due to an interaction between guest and host atoms and with quasi-pairing of the guest atoms along their chain. Recently, it has been 
shown that the commensurate model with 32 atoms supercell model and $\mathrm{c}_{\text {host }} / \mathrm{c}_{\text {guest }}=4 / 3$ describes approximately the modulation of the experimental crystal structure. ${ }^{67}$ There should be some effects of the modulated structure on the lattice dynamics, which is not taken into account in the simplified model and give several Raman-active modes due to the guest Bi chain. The decomposition of the vibrational irreducible representations of the Bi-IV phase with 8 atoms/unit cell is: $\Gamma=3 \mathrm{~A}_{\mathrm{g}} \oplus 3 \mathrm{~B}_{1 \mathrm{~g}} \oplus 3 \mathrm{~B}_{2 \mathrm{~g}} \oplus 3 \mathrm{~B}_{3 \mathrm{~g}} \oplus 2 \mathrm{~A}_{\mathrm{u}} \oplus 4 \mathrm{~B}_{1 \mathrm{u}} \oplus 4 \mathrm{~B}_{2 \mathrm{u}} \oplus 2 \mathrm{~B}_{3 \mathrm{u}} . \mathrm{B}_{1 \mathrm{u}}, \mathrm{B}_{2 \mathrm{u}}$ and $B_{3 u}$ are the acoustic modes, there are 12 Raman-active modes of $A_{g}, B_{1 g}, B_{2 g}$ and $B_{3 g}$ symmetries and 7 infrared-active modes of $B_{1 u}, B_{2 u}$ and $B_{3 u}$ symmetries.

For the body-centered cubic $\mathrm{V}$ structure with 1 atom/unit cell, we have the following decomposition of the vibrational irreducible representations: $\Gamma=\mathrm{F}_{\mathrm{u}}(\mathrm{x}, \mathrm{y}, \mathrm{z})$. In this case, the mode with $F_{u}$ symmetry is the acoustic mode and there is neither Raman-active nor infraredactive modes.

The phonon dispersion curves of the different phases are shown in the Figures 5 and 6 for room pressure and for different pressures. There are vibrational modes with negative wave numbers for the Bi-IV and $\mathrm{Bi}-\mathrm{V}$ phases at $0 \mathrm{GPa}$. This means that these phases are dynamically unstable at room pressure. However, when increasing the pressure, they become dynamically stable. In the case of the $\mathrm{Bi}-\mathrm{I}, \mathrm{Bi}-\mathrm{II}$ and $\beta-\mathrm{Bi}$, pressure has little effect on the acoustical branches, especially at the vicinity of the Brillouin zone center. In the case of Bi-I, the frequencies of the optical modes around the Brillouin zone center decrease with increasing pressure, meaning that they have negative Grüneisen parameters, in agreement with the prior Raman experiments ${ }^{58}$ and our own Raman scattering experiments (see below). For the other phases, the frequencies of the optical modes increase with pressure, as expected for positive Grüneisen parameters. The phonon DOS of the different phases are shown in the Figure 7 for room pressure and for different pressures. In the case of the Bi-I phase, there is a gap between the acoustic phonons and the optical phonons and of up to almost $120 \mathrm{~cm}^{-1}$. In the case of the high pressure phases, 
the phonon DOS spread out up to $100 \mathrm{~cm}^{-1}$, except for the Bi-III phase for which this is up to $110 \mathrm{~cm}^{-1}$. As can be seen in Fig. 7, our results agree well with those of Khasanov et al ${ }^{44}$ concerning the Bi-III phase at $2.5 \mathrm{GPa}$. We do not confirm the presence of the additional phason at low energy in this structure, which was found by Brown et al. ${ }^{43}$

In order to probe the vibrational properties of the guest-host Bi-III structure, we report its atomprojected phonon DOS at $5 \mathrm{GPa}$ in the Figure 8. Between 22 and $45 \mathrm{~cm}^{-1}$ and between 60 and $100 \mathrm{~cm}^{-1}$, the host atoms are the main contribution of the phonon DOS. There is a significant contribution from the motions of the guest atoms along the c direction at about $20 \mathrm{~cm}^{-1}$ and this is the main contribution for the peak at about $105-110 \mathrm{~cm}^{-1}$. At about $20 \mathrm{~cm}^{-1}$, the two infraredactive modes of symmetry $\mathrm{A}_{2 \mathrm{u}}\left(18.5 \mathrm{~cm}^{-1}\right)$ and $\mathrm{E}_{\mathrm{u}}\left(21 \mathrm{~cm}^{-1}\right)$ and the lowest Raman-active mode of symmetry $\mathrm{A}_{1 \mathrm{~g}}\left(22.3 \mathrm{~cm}^{-1}\right)$ have a significant contribution from the guest atoms in the $a b$ plane for the $\mathrm{E}_{\mathrm{u}}$ mode and in the $c$ direction for the $\mathrm{A}_{2 \mathrm{u}}$ and $\mathrm{A}_{1 \mathrm{~g}}$ modes. The four highest energy modes with $\mathrm{A}_{1 \mathrm{u}}, \mathrm{A}_{2 \mathrm{u}}, \mathrm{A}_{1 \mathrm{~g}}$ and $\mathrm{A}_{2 \mathrm{~g}}$ symmetries at about $105 \mathrm{~cm}^{-1}$ arise mainly from motions of the guest atoms in the $c$ direction. Therefore, the lowest and the highest energy Raman-active modes of $\mathrm{A}_{1 \mathrm{~g}}$ symmetry involve mainly motion of guest atoms along the $c$ direction.

Gorman et al. recently showed that $\beta$-Bi and Bi-II are closely related by a shear. ${ }^{40}$ From the group-subgroup relation between the $\mathrm{I}_{1} /$ amd space group of $\beta$-Bi and the $\mathrm{C} 2 / \mathrm{m}$ space group of $\mathrm{Bi}-\mathrm{II}$ and because the size of the cell is not doubled, there could be a displacive phase transition between these two structures and that is driven by the $\mathrm{E}_{\mathrm{g}}$ optical phonon mode. ${ }^{71}$ When looking our calculations for the $\beta-\mathrm{Bi}$, the frequency of the $\mathrm{E}_{\mathrm{g}}$ optical phonon mode is $76.4 \mathrm{~cm}^{-1}$ at $0 \mathrm{GPa}$ and increases to $87.7 \mathrm{~cm}^{-1}$ at $2.5 \mathrm{GPa}$. Because of these large values and the observed hardening of this mode, we do not believe that there will be a displacive phase transition between the $\beta$ $\mathrm{Bi}$ and the Bi-II.

The evolution of Raman spectra of $\mathrm{Bi}$ as function of pressure was shown in Figure 9 under nonhydrostatic conditions. We use such conditions as direct contact of the powder sample with the 
diamond anvil leads to the dissipation of the laser heating and higher laser power can be used, hence obtain better signal for the high pressure phases and especially the Bi-III phase than using hydrostatic conditions. Raman modes of $\mathrm{Bi}-\mathrm{I}\left(\mathrm{E}_{\mathrm{g}}\right.$ and $\left.\mathrm{A}_{1 \mathrm{~g}}\right)$ were observed below $3 \mathrm{GPa}$ at about $70 \mathrm{~cm}^{-1}$ and $97 \mathrm{~cm}^{-1}$ at room temperature. Transformation from Bi-I to Bi-II occurs at $3.04 \mathrm{GPa}$ and phase mixtures were also observed. The low-frequency $\mathrm{A}_{1 \mathrm{~g}}$ mode of $\mathrm{Bi}$-II was observed up to 3.60 GPa. Bi-III Raman modes appeared at $3.60 \mathrm{GPa}$ in the spectrum of II-III phase mixtures and exhibit broad Raman features A, B and C with weak intensities, maybe because of the large number of Raman modes $\left(\mathrm{A}_{1 \mathrm{~g}}, \mathrm{E}_{\mathrm{g}}, \mathrm{B}_{1 \mathrm{~g}}, \mathrm{~B}_{2 \mathrm{~g}}\right)$ and structural imperfections. A significant modification of the spectrum was observed at 9.17 GPa and Raman modes were absent due to the bcc structure of $\mathrm{Bi} \mathrm{V}$. In addition, the Raman spectrum of Bi-II was observed after the pressure was decreased to $2.7 \mathrm{GPa}$, indicating that the phase transition was reversible upon decompression.

In Figure 10, we report the experimental spectra at $3 \mathrm{GPa}$ and $5 \mathrm{GPa}$. At $3 \mathrm{GPa}$, there is a coexistence of the Raman modes of the Bi-I and Bi-II phases. The positions of the Raman active modes of the Bi-II phase agree well with the prior results of Olijnyk et al. ${ }^{58}$ By comparing our experimental results with our DFT calculations (at $2.5 \mathrm{GPa}$ ), one can assign the different Raman-active modes of Bi-II phase.

Figure 11 shows the Raman shifts of different phases as function of pressure under quasihydrostatic and non-hydrostatic conditions. It can be observed from scatter of the frequency data that the effect pressure is reversible. Phases transitions are indicated by discontinuous line. We can see some difference between the two experimental conditions. At room temperature and pressure, Raman modes of Bi-I were observed at $70.7 \mathrm{~cm}^{-1}$ and $97.8 \mathrm{~cm}^{-1}$. This in good agreement with our DFT calculations for which one finds at $0 \mathrm{GPa}$ the vibrational frequencies of 71 and $99.7 \mathrm{~cm}^{-1}$ for the $A_{1 g}$ and $E_{g}$ modes. This agrees reasonably well with prior calculations ${ }^{48-54}$ and experiments. ${ }^{55-58}$ Then, with increasing the pressure and under the two 
experimental conditions, Raman modes of $\mathrm{Bi}-\mathrm{I}$ phase $\left(\mathrm{E}_{\mathrm{g}}\right.$ and $\left.\mathrm{A}_{1 \mathrm{~g}}\right)$ shift towards globally the lower wavenumber under quasi-hydrostatic conditions and they move slightly to the higher wavenumber under non-hydrostatics conditions.

In prior experiment works under quasi-hydrostatic conditions, large decreases were observed for the frequencies of the Raman modes of Bi-I phase with pressure. ${ }^{58}$ Similar large decreases of the frequencies of the Raman modes are observed in our DFT calculations (see Table 1). This gives negative mode Grüneisen parameters for these Raman modes. Using the bulk modulus from Degtyareva et al., ${ }^{70}$ we can calculate the experimental mode Grüneisen parameters $\gamma_{i}$ of the Raman modes of Bi-I phase using either our data or those of Olyjnik et al ${ }^{58}$ (see Table 2). The Grûneisen parameter is larger for the $\mathrm{E}_{\mathrm{g}}$ mode than for the $\mathrm{A}_{1 \mathrm{~g}}$ mode, as in the case of rhombohedral $\mathrm{Sb}$ and $\mathrm{As}{ }^{58,69,72}$ The mode Grüneisen parameters $\gamma_{\mathrm{i}}$ of Bi-I are larger than those found by Olyjnik et al ${ }^{58}$ for rhombohedral $\mathrm{Sb}$, but of the same order of magnitude than those found by Wang et al ${ }^{69}$ for rhombohedral $\mathrm{Sb}$, who find a non-linear pressure variation of the frequencies of the Raman modes. The mode Grüneisen parameters $\gamma_{\mathrm{i}}$ of Bi-I are slightly smaller than those found for rhombohedral As. ${ }^{72}$

In contrast, Raman modes for both Bi II and III move to the higher wavenumber under both experimental conditions as well as in the DFT calculations, meaning they have positive mode Grüneisen parameters. One can see a good correspondence between our DFT calculations and the Oljinyk's experiments for the Bi-II phase. ${ }^{58}$ For the Bi-III phase, under quasi-hydrostatic (non-hydrostatic) conditions, the peak B at about $70 \mathrm{~cm}^{-1}$ upshifts by $1.5 \pm 0.2(2 \pm 0.4) \mathrm{cm}^{-}$ ${ }^{1} / \mathrm{GPa}$ and the peak $\mathrm{C}$ at about $90 \mathrm{~cm}^{-1}$ upshifts by $1.7 \pm 0.2(1.1 \pm 0.4) \mathrm{cm}^{-1} / \mathrm{GPa}$. For the peak of the calculated phonon DOS at $88 \mathrm{~cm}^{-1}$, one finds $\Delta \omega / \Delta \mathrm{P}=2.66 \mathrm{~cm}^{-1} / \mathrm{GPa}$, about 2 times the experimental values. Using the bulk modulus of Bi-III from Degtyareva et al., ${ }^{70}$ we can calculate the experimental mode Grüneisen parameters $\gamma_{i}$ of the Raman modes of Bi-III phase. We find, under quasi-hydrostatic (non-hydrostatic) conditions, $\gamma_{i}=1.14$ (1.51) for the peak B 
and $\gamma_{i}=1(0.65)$ for the peak $\mathrm{C}$. These values are lower than the mode Grüneisen parameters found by Wang et al. ${ }^{69}$ for the Sb-II phase, which is isostructural with the Bi-III phase. The low-energy mode at about $28 \mathrm{~cm}^{-1}$ of $\mathrm{Bi}$ III, which corresponds to $\mathrm{E}_{\mathrm{g}}^{2}$ by comparison with the DFT calculations, was observed only for the powder sample under non-hydrostatic conditions (no PTM), but was not intense enough to be observed in the argon PTM. Its experimental position upshifts by $0.72 \pm 0.05 \mathrm{~cm}^{-1} / \mathrm{GPa}$, which is in good agreement with the DFT calculations $\left(\Delta \omega / \Delta \mathrm{P}=0.6 \mathrm{~cm}^{-1} / \mathrm{GPa}\right)$. The experimental mode Grüneisen of this low energy Raman mode $\gamma_{\mathrm{i}}$ is 1.36 . The position of the peak at $70 \mathrm{~cm}^{-1}$ increases linearly with pressure for both experimental conditions, whereas the peak at $90 \mathrm{~cm}^{-1}$ exhibits almost no change with nonhydrostatic pressure and increases almost linearly under increasing quasi-hydrostatic pressure. Thus, the non-hydrostatic conditions modify the pressure dependence of the Raman modes. Moreover, we still observed some peaks of phase II at 3.6 GPa under quasi-hydrostatic conditions, indicating that low-pressure phases became metastable at higher pressure due to nonhydrostaticity.

\section{Conclusion}

DFT calculations were performed in order to study the stability and pressure dependence of the different crystal structures of Bi and of their lattice dynamics. We have also determined the assignment of the symmetry of the Raman modes of $\mathrm{Bi}-\mathrm{I}$ and $\mathrm{Bi}-\mathrm{II}$ phases and their pressure dependence compared well with the experimental results. The Raman spectrum of Bi-III phase was measured for the first time and can be interpreted based on the DFT calculations. From the DFT calculations, we have determined the vibrational modes due to motion of the guest atoms along the $c$ direction. 


\section{References}

${ }^{1}$ Lenoir, B. ; Scherrer, H. ; Caillat, T., An Overview of Recent Developments for BiSb Alloys. Semicond. Semimet. 2001, 69, 101-137.

${ }^{2}$ Kim, J. ; Shim, W. ; Lee, W., Bismuth nanowire thermoelectrics. J. Mater. Chem. C 2015, 3, 11999-12013.

${ }^{3}$ Cannon, J. F., Behavior of the Elements at High Pressures.J. Phys. Chem. Ref. Data 1974, 3, 781-824.

${ }^{4}$ Cucka, P.; Barrett, C. S., The Crystal Structure of Bi and of Solid Solutions of Pb, Sn, Sb and Te in Bi. Acta Crystallogr. 1962, 15, 865-872.

${ }^{5}$ Akselrud, L. G. ; Hanfland, M. ; Schwarz, U., Refinement of the crystal structure of Bi-II at 2.54 GPa, Z. Kristallogr. NCS 2003, 218, 415-416.

${ }^{6}$ Principi, E. ; Minicucci, M. ; Di Cicco, A. ; Trapananti, A. ; De Panfilis, S. ; Poloni, R., Metastable phase diagram of Bi probed by sinfle-energy x-ray detection absorption and angular dispersive x-ray diffraction. Phys. Rev. B 2006, 74, 064101.

${ }^{7}$ Chaimayo, W. ; Lundergaard, L. F. ; Loa, I. ; Stinton, G. W. ; Lennie, A. R. ; McMahon, M. I., High-pressure, high-temperature single-crystal study of Bi-IV. High Press. Res. 2012, 32, 442-449.

${ }^{8}$ Degtyareva, O. ; McMahon, M.I. ; Nelmes, R.J, Crystal Structure of the High Pressure Phase of Bismuth Bi-III. Mater. Sci. Forum 2001, 378-381, 469-475.

${ }^{9}$ Haussermann, U. ; Soedelberg, K. ; Norrestam, R., Comparative Study of the High-Pressure of As, Sb, and Bi. J. Am. Chem. Soc. 2002, 124, 15359-15367.

${ }^{10}$ Schaufelberger, Ph. ; Merx, H. ; Contré, M. Structure crystalline du bismuth V. High Temp.High Pressures 1972 4, 221-230.

${ }^{11}$ Brugger, R. M. ; Bennion, R. B. ; Worlton, T. G., The crystal structure of bismuth II at 26 kbar Phys. Lett. A 1967, 24, 714-717. 
${ }^{12}$ Kabalkina, S. S. ; Kolobyanina, T. N. ; Vereshchagin, L. F., Investigation of the crystal structure of the antimony and the bismuth high pressure phases. Sov. Phys. JETP 1970, 31, 259263.

${ }^{13}$ Duggin, M. J., A high pressure phase in arsenic and its relation to pressure-induced phase changes in group 5B elements. J. Phys. Chem. Solids 1972, 33, 1267-1271.

${ }^{14}$ Fedotov, V. K. ; Ponyatovskii, E. G. ; Somenkov, V., Neutronographical study of bismuth to 30 kbar pressure. Sov. Phys. Solid State 1978, 20, 628-636.

${ }^{15}$ Chen, J. H. ; Iwasaki, H. ; Kikegawa, T., Crystal structure of the high-pressure phases of bismuth Bi III and Bi III' by high energy synchrotron x-ray diffraction. High Press. Res. 1996, $15,143-158$.

${ }^{16}$ McMahon, M. I. ; Degtyareva, O. ; Nelmes, R. J., Ba-IV-Type Incommensurate Crystal Structure in Group-V Metals. Phys. Rev. Lett. 2000, 85, 4896-4899.

${ }^{17}$ Giardini, A. A. ; Samara, G. A.,The compressibility of bismuth and its upper transition pressure J. Phys. Chem. Solids 1965, 26, 1523-1528, and references therein.

18 McMahon, M. I. ; Degtyareva, O. ; Nelmes, R. J. ; van Smaalen, S. ; Palatinus, L., Incommensurate modulations of Bi-III and Sb-II. Phys. Rev. B 2007, 75, 184114.

${ }^{19}$ Akahama, Y.; Kawamura, H. ; Singh, A. K., Equation of states of bismuth to $222 \mathrm{GPa}$ and comparison of gold and platinum pressure scales to 145 GPa. J. Appl. Phys. 2002, 92, 58925897.

${ }^{20}$ Getting, I. C., New determination of the Bi I-II equilibrium pressure: a proposed modification to the practical pressure scale. Metrologia, 1998, 35, 119-132.

${ }^{21}$ Bundy, F. P., Phase Diagram of Bismuth to $130000 \mathrm{~kg} / \mathrm{cm}^{2}, 500^{\circ} \mathrm{C}$. Phys. Rev. 1958, 110, 314-318.

22 Phase Transformations of Elements Under High Pressure, Eds. Tonkov, E. Yu ; Ponyatovsky, E. G., CRC Press, Boca Raton, 2005, pp. 148-157. 
${ }^{23}$ Aoki, K. ; Fujiwara, S. ; Kusakabe, M., Stability of the bec Structure of Bismuth at High Pressure. J. Phys. Soc. Jpn. 1982, 51, 3826-3830.

${ }^{24}$ Bridgman, P. W., Polymorphism, Principally of the Elements, up to 50,000 kg/cm². Phys. Rev. 1935, 48, 893-906.

${ }^{25}$ Klement, W. ; Kennedy, G. C. ; Jayaraman, A., Phase Diagrams of Arsenic Antimony, and Bismuth at Pressures up to 70 kbars. Phys. Rev. 1963, 131, 632-637.

${ }^{26}$ Kossowsky, R., Observations of Bi 3-4 and $\mathrm{Bi} 4-\mathrm{V}$ transitions by resistivity measurements. Trans. Metall. Soc. AIME 1966, 236, 1746.

${ }^{27}$ Nichols, S., The pressure-induced Bi III-IV transition. J. Phys. D 1971, 4, 783-786.

${ }^{28}$ Nichols, S., A bismuth triple point at $41 \mathrm{kbar}, 175^{\circ}$ C. J. Phys. D 1972, 5, 799-802.

${ }^{29}$ Nichols, S., The volume change associated with the modification of bismuth III at $42 \mathrm{kbar}$ and $20^{\circ}$ C. J. Phys. D 1972, 5, 1898-1901.

${ }^{30}$ Homan, C. G., Phase diagram of bismuth up to 140 kbars. J. Phys. Chem. Solids 1975, 36, 1249-1254.

${ }^{31}$ Lotter, N. ; Wittig, J., Evidence for an Electronic Phase Transition in Bismuth under Pressure. Europhys. Lett. 1988, 6, 659-662.

${ }^{32}$ Yoneda A. ; Endo, S., Phase transitions in barium and bismuth under high pressure, J. Appl. Phys. 1980, 51, 3216-3221.

${ }^{33}$ Wang, Z. ; Liu, Y. ; Bi, Y. ; Song, W. ; Xie, H., Hydrostatic pressure and temperature calibration based on phase diagram of bismuth., High Press. Res. 2012, 32, 167-175.

${ }^{34}$ Chen, H.-Y. ; Xiang, S.-K. ; Yan, X.-Z. ; Zheng, L.-R. ; Zhang, Y. ; Liu, S.-G. ; Bi, Y., Phase transition of solid bismuth under high pressure. Chin. Phys. B 2016, 25, 108103.

35 Chen, J. H. ; Iwasaki, H. ; Kikegawa, T., Structural study of the high-pressure-hightemperature phase of bismuth using high energy synchrotron radiation. J. Phys. Chem. Solids 1997, 58, 247-255. 
${ }^{36}$ Degtyareva, V. F., Crystal structure of high-pressure phase in Bi-based alloys related to Si VI. Phys. Rev. B 2000, 62, 9-12.

37 Tikhomirova, N. A. ; Tonkov, E. Yu. ; Stishov, S. M., New phase of bismuth. JETP Lett. 1966, 3, 60-62.

${ }^{38}$ Lin, C. ; Smith, J. S. ; Sinogeikin, S. V. ; Kono, Y. ; Park, C. ; Kenney-Bensen, C. ; Shen, G., A metastable liquid melted from a crystalline solid under decompression. Nat. Commun. 2017, $8,14260$.

39 Lin, C. ; Smith, J. S. ; Sinogeikin, S. V. ; Shen, G., Effect of stress on melting of rhombohedral bismuth. Appl. Phys. Lett. 2017, 110, 161904.

${ }^{40}$ Gorman, M. G. ; Coleman, A. L. ; Briggs, R. ; McWilliams, R. S. ; Herrmann, A. ; McGonegle, D. ; Bolme, C. A. ; Gleason, A. E. ; Galtier, E. ; Lee, H. J. ; Granados, E. ; McBride, E. E. ; Rothman, S. ; Fratanduono, D. E. ; Smith, R. F. ; Collins, G. W. ; Eggert, J. H. ; Wark, J. S. ; McMahon, M. I., Recovery of metastable dense Bi synthesized by shock compression. Appl. Phys. Lett. 2019, 114, 120601.

${ }^{41}$ Pépin, C. M. ; Sollier, A. ; Marizy, A. ; Occelli, F. ; Sander, M. ; Torchio, R. ; Loubeyre, P., Kinetics and structural changes in dynamically compressed bismuth. Phys. Rev. B 2019, 100, 060101.

${ }^{42}$ Gorman, M. G. ; Coleman, A. L. ; Briggs, R. ; McWilliams, R. S. ; McGonegle, D. ; Bolme, C. A. ; Gleason, A. E. ; Galtier, E. ; Lee, H. J. ; Granados, E. ; Sliwa, M. ; Sanloup, C. ; Rothman, S. ; Fratanduono, D. E. ; Smith, R. F. ; Collins, G. W. ; Eggert, J. H. ; Wark, J. S. ; McMahon, M. I., Femtosecond diffraction studies of solid and liquid phase changes in shockcompressed bismuth. Scient. Rep. 2018, 8, 16927.

${ }^{43}$ Brown, P. ; Semeniuk, K. ; Wang, D. ; Montserrat, B. ; Pickard, C. J. ; Grosche, M. F., Strong coupling superconductivity in a quasi-periodic host-guest structure, Sci. Adv. 2018, 4, 4793. 
${ }^{44}$ Khasanov, R. ; Radonjic, M. M. ; Luetkens, H. ; Morenzoni, E. ; Simutis, G. ; Schönecker, S. ; Östlin, A. ; Chioncel, L. ; Amato, A., Superconductivity of Bi-III phase of elemental bismuth : Insights from muon-spin rotation and density functional theory. Phys. Rev. B 2018, 98, 140504.

${ }^{45}$ Khasanov, R. ; Luetkens, H. ; Morenzoni, E. ; Simutis, G. ; Schönecker, S. ; Appelt, W. H. ; Östlin, A. ; Chioncel, L. ; Amato, A., Superconducting nature of the Bi-II phase of elemental bismuth.Phys. Rev. B 2019, 99, 174506.

${ }^{46}$ Clarke, S. M. ; Amsler, M. ; Walsh, J. P. S. ; Yu, T. ; Wang, Y.B. ; Meng, Y. ; Jacobsen, S. D. ; Wolverton, C. ; Freeman, D. E., Creating Cu-Bi Compounds via High-Presure Synthesis: A Combined Experimental and Theoretical Study. Chem. Mater. 2017, 29, 5276-5285.

${ }^{47}$ Amsler, M. ; Hegde, V. I. ; Jacobsen, S. D. ; Wolverton, C., Exploring the High-Pressure Materials Genome. Phys. Rev. X 2018, 8, 041021.

${ }^{48}$ Diaz-Sanchez, L. E. ; Romero, A. H. ; Gonze, X., Phonon band structure and interatomic force constants for bismuth : Crucial role of spin-orbit interaction. Phys. Rev. B 2007, 76, 104302.

${ }^{49}$ Murray, E. D. ; Fahy, S. ; Prendergast, D. ; Ogitsu, T. ; Fritz, D. M. ; Reis, D. A., Phonon dispersion relations and softening in photoexcited bismuth from first principles. Phys. Rev. B 2007, $75,184301$.

${ }^{50}$ Yang, J. ; Huang, G. Q. ; Zhu, X. F., Thickness evolution of phonon properties in ultrathin Bi (111) films. phys. Stat. sol. (b) 2013, 250, 1937-1942.

${ }^{51}$ Lee, S. ; Esfarjani, K. ; Mendoza, J. ; Dresselhaus, M. S. ; Chen, G., Lattice thermal conductivity of Bi, Sb, and Bi-Sb alloy from first principles. Phys. Rev. B 2014, 89, 085206.

52 Alcantara-Ortigoza, M. ; Sklyadneva, I. Y. ; Heid, R. ; Chulkov, E. V. ; Rahman, T. S. ; Bohnen, K.-P. ; Echenique, P. M., Ab initio lattice dynamics and electron-phonon coupling of Bi(111). , Phys. Rev. B 2014, 90, 195438. 
${ }^{53}$ Arnaud, B. ; Lebègue, S. ; Raffy, G., Anisotropic thermal expansion of bismuth from first principles. Phys. Rev. B 2016, 93, 094106.

${ }^{54}$ Markov M. ;Sjaskte, J. ; Fugallo, G. ; Paulatto, L. ; Lazzeri, M. ; Mauri, F. ; Vast, N., Nanoscale mechanisms for the reduction of heat transport in bismuth., Phys. Rev. B 2016, 93, 064301.

${ }^{55}$ Mukherjee, D. ; Sahoo, B. D. ; Joshi, K. D. ; Gupta, S. C., On equation of state, elastic, and lattice dynamics stabilitu of bcc bismuth under high presure: Ab-initio calculations. J. Appl. Phys. 2014, 115, 053702.

55 Zitter, R. N., in: Physics of Semimetals and Narrow Gap Semiconductors, eds D. L. Carter and R. T. Bate, Pergamon, New York, 1971, pp. 285-287.

${ }^{56}$ Grant, W. B. ; Schulz, H. ; Hüfner, S. ; Pelzl, J., Investigation of k = 0 Phonons in Bi, Cd, Mg, and Zn by Raman scattering. Phys. Stat. Sol. (b) 1973, 60, 331-340.

${ }^{57}$ Richter, W. ; Fjeldly, T. ; Renucci, J. ; Cardona, M., Hydrostatic pressure dependence of the zone-center phonons in group $\mathrm{Vb}$ Materials: $\mathrm{As}, \mathrm{Sb}, \mathrm{Bi}$. In Proceedings of the International Conference on Lattice Dynamics, ed. M. Balkanski, Flammarion Sciences, Paris, 1978, pp. 104106.

${ }^{58}$ Olijnyk, H. ; Nakano, S. ; Takemura, K., First- and second order Raman scattering in Sb and Bi at high pressure. Phys. Stat. Sol. (b) 2007, 244, 3572-3582.

${ }^{59}$ Piermiarini, G. J. ; Block, S. ; Barnett, J. D. ; Forman, R. A., Calibration of the pressure dependence of the $\mathrm{R}_{1}$ ruby fluorescence line to 195 kbars, J. Appl. Phys. 1975, 46, 2774-2780. ${ }^{60}$ Kresse, G. ; Furthmüller, J., Efficient iterative schemes for ab initio total-energy calculations using a plane-wave basis set. Phys. Rev. B 1996, 54, 11169-11186.

${ }^{61}$ Kresse, G.; Joubert, D., From ultrasoft pseudopotentials to the projector augmented-wave method. Phys. Rev. B 1999, 59, 1758-1775. 
${ }^{62}$ Perdew, J. P.; Burke, K.; Erzenhof, M., Generalized Gradient Approximation Made Simple. Phys. Rev. Lett. 1996, 77, 3865-3868.

${ }^{63}$ Methfessel, M. ; Paxton, A.T., High-precision sampling for Brillouin-zone integration in metals, Phys. Rev. B 1989, 40, 3616-3621.

${ }^{64}$ Parlinski, K.; Li, Z.-Q.; Kawazoe, Y., First-Principles Determination of the Soft Mode in cubic $\mathrm{ZrO}_{2}$. Phys. Rev. Lett. 1997, 78, 4063-4066.

${ }^{65}$ Blöchl, P. E. ; Jepsen, O. ; Andersen, O. K., Improved tetrahedron method for Brillouin-zone integrations, Phys. Rev. B 1994, 49, 16223-16233.

${ }^{66}$ Schifferl, D. ; Barrett, C. S., The crystal of Arsenic at 4.2, 78 and 299 K. J. Appl. Crystallogr. 1969, 2, 30-36.

${ }^{67}$ Kartoon, D. ; Makov, G., Structural and electronic properties of the incommensurate hostguest Bi-III phase. Phys. Rev. B 2019, 100, 014104.

${ }^{68}$ Yoon, W. ; Paik, J. S. ; LaCourt, D. ; Perepezko, J. H., The effect of pressure on phase selection during nucleation in undercooled bismuth. J. Appl. Phys. 1986, 60, 3489-3494.

${ }^{69}$ Wang, X. ; Kunc, K. ; Loa, I. ; Schwarz, U. ; Syassen, K., Effect of pressure on the Raman modes of antimony. Phys. Rev. B 2006, 74, 134305.

${ }^{70}$ Degtyareva, O. ; McMahon, M. I. ; Nelmes, R. J., High pressure structural studies of goup15 elements. High Press. Res. 2004, 24, 319-356.

${ }^{71}$ Stokes, H. T.; Hatch, D. M., Isotropy subgroups of the 230 crystallographic space groups, World Scientific Publishing Co. Pte. Ltd, 1988.

${ }^{72}$ Beister, H. J. ; Strössner, K. ; Syassen, K., Rhombohedral to simple-cubic phase transition in arsenic under pressure. Phys. Rev. B 1990, 41, 5535-5543. 


\section{Table captions}

Table 1: Frequencies of the Raman active modes and their pressure dependence for the Bi-I phase from our DFT calculations, experiments (hydrostatic and non-hydrostatic conditions) and Oljinyk’s experiments. ${ }^{58}$

Table 2: Frequencies of the Raman active modes, their pressure dependence and their Grüneisen parameters $\gamma_{i}$ for the Bi-II phase from our DFT calculations, experiments (hydrostatic and non-hydrostatic conditions) and Oljinyk’s experiments. ${ }^{58}$ 
Tables

\begin{tabular}{|c|ccc|} 
Mode symmetry & $\mathrm{Ag}^{1}$ & $\mathrm{Ag}^{2}$ & $\mathrm{~B}_{\mathrm{g}}$ \\
\hline$\omega\left(\mathrm{cm}^{-1}\right) \mathrm{DFT}(2.5 \mathrm{GPa})$ & 44.7 & 76.3 & 81.9 \\
$\Delta \omega / \Delta \mathrm{P}\left(\mathrm{cm}^{-1} / \mathrm{GPa}\right) \mathrm{DFT}$ & 0.64 & 4.72 & 4.72 \\
$\omega\left(\mathrm{cm}^{-1}\right)$ exp. non-hydro $(3.04 \mathrm{GPa})$ & 39.7 & -- & 83.3 \\
$\omega\left(\mathrm{cm}^{-1}\right)$ exp. hydro $(2.92 \mathrm{GPa})$ & $38.7 \pm 0.50$ & -- & 83.7 \\
$\Delta \omega / \Delta \mathrm{P}\left(\mathrm{cm}^{-1} / \mathrm{GPa}\right)$ exp, non-hydro & -0.13 & & 0.43 \\
$\Delta \omega / \Delta \mathrm{P}\left(\mathrm{cm}^{-1} / \mathrm{GPa}\right)^{58}$ & 0.82 & & 4.39
\end{tabular}

Table 1

\begin{tabular}{|c|cc|} 
Mode symmetry & $\mathrm{E}_{\mathrm{g}}$ & $\mathrm{A}_{1 \mathrm{~g}}$ \\
\hline$\omega\left(\mathrm{cm}^{-1}\right) \mathrm{DFT}(0 \mathrm{GPa})$ & 71 & 99.7 \\
$\Delta \omega / \Delta \mathrm{P}\left(\mathrm{cm}^{-1} / \mathrm{GPa}\right) \mathrm{DFT}$ & -3.53 & -1.51 \\
$\omega\left(\mathrm{cm}^{-1}\right) \exp (0 \mathrm{GPa})$ & 70.7 & 97.8 \\
$\Delta \omega / \Delta \mathrm{P}\left(\mathrm{cm}^{-1} / \mathrm{GPa}\right)$ exp, non-hydro & $0.21 \pm 0.16$ & $0.45 \pm 0.12$ \\
$\Delta \omega / \Delta \mathrm{P}\left(\mathrm{cm}^{-1} / \mathrm{GPa}\right)$ exp, hydro & $-1.29 \pm 0.03$ & $-0.2 \pm 0.2$ \\
$\gamma_{\mathrm{i}} \exp$, hydro & -0.7 & -0.08 \\
$\Delta \omega / \Delta \mathrm{P}\left(\mathrm{cm}^{-1} / \mathrm{GPa}\right){ }^{58}$ & -1.67 & -0.75 \\
$\gamma_{\mathrm{i}}{ }^{58}$ & -0.98 & -0.31
\end{tabular}

Table 2 


\section{Figure captions}

Figure 1: Crystal structures of Bi under pressure: (a) Bi-I: rhombohedral A7 structure ${ }^{4}$; (b) Bi-II: monoclinic structure ${ }^{5}$; (c) $\beta$-Bi or Bi-II': body-centered tetragonal structure ${ }^{6}$; (e) BiIV: C-centered orthorhombic structure ${ }^{7}$; (e) Bi-III: tetragonal host-guest structure ${ }^{8,9}$; (f) Bi$\mathrm{V}$ : body-centered cubic structure ${ }^{10}$

Figure 2: (top) Volume normalized to the volume of rhombohedral $\mathrm{Bi}$ at $0 \mathrm{GPa}\left(\mathrm{V}_{0}\right)$ as a function of the pressure for the different high-pressure structures of bismuth calculated from the DFT (different lines) and compared to experimental volumes (symbols); ${ }^{5,16}$ (bottom) energy in function of the pressure for the different high-pressure structure of bismuth calculated from the DFT.

Figure 3: (left) Gibbs energy of formation $\Delta \mathrm{G}$ of $\mathrm{Bi}-\mathrm{I}, \mathrm{Bi}-\mathrm{II}, \beta-\mathrm{Bi}$ and $\mathrm{Bi}-\mathrm{III}$ at $2.5 \mathrm{GPa}$ as a function of the temperature; (right) difference of Gibbs energy of Bi-III and Bi-IV at $5 \mathrm{GPa}$ as a function of the temperature.

Figure 4: (a) Electronic density of states of Bi-II at $2.5 \mathrm{GPa}$; (b) electronic density of states of $\beta$-Bi at $2.5 \mathrm{GPa}$; (c) electronic density of states of Bi-III at $5 \mathrm{GPa}$; (d) electronic density of states of Bi-IV at $5 \mathrm{GPa}$.

Figure 5: Phonon dispersion curves of Bi-I, Bi-II, $\beta$-Bi and Bi-V phases.

Figure 6: Phonon dispersion curves of Bi-III and Bi-IV phases.

Figure 7: Phonon density of states of Bi-I, Bi-II, $\beta$-Bi, Bi-III, Bi-IV and Bi-V phases. 
Figure 8: Atom-projected phonon density of states of Bi-III.

Figure 9: Raman spectra of bismuth at different pressure under non-hydrostatic conditions.

Figure 10: Raman spectra of bismuth at $3 \mathrm{GPa}$ and $5 \mathrm{GPa}$ under non-hydrostatic conditions. The bars correspond to the position of the calculated Raman-active modes for the Bi-I and BiII phases at $2.5 \mathrm{GPa}$ and for the Bi-III phase at $5 \mathrm{GPa}$

Figure 11: Positions of the Raman lines of bismuth in function of pressure for powder under non-hydrostatic conditions (left) and for Bi pieces in argon, i. e. under quasi-hydrostatic conditions (right). The filled symbols correspond to measurement under compression whereas the hollow symbols correspond to measurement under decompression. 
Figures
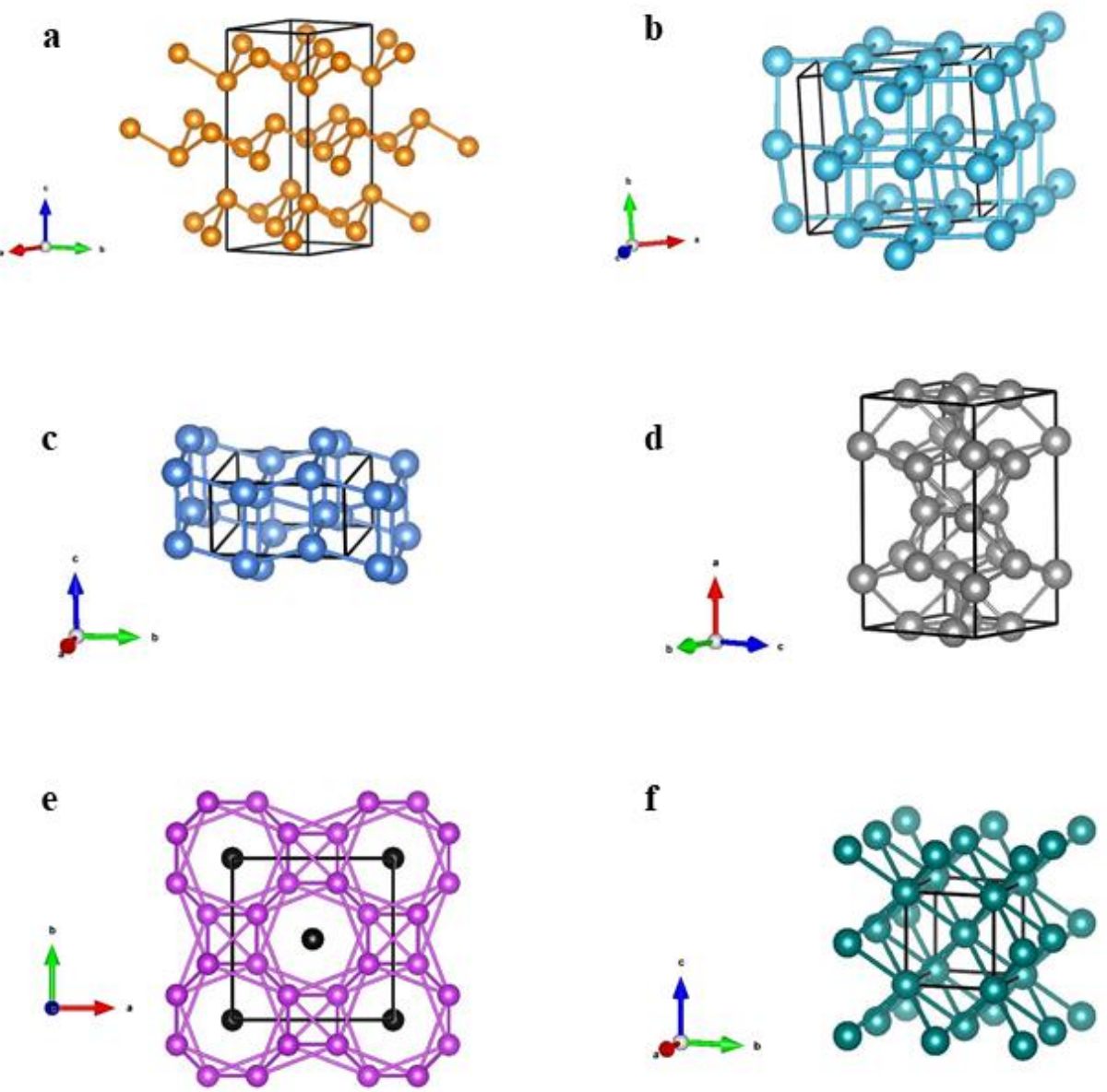

Figure 1 


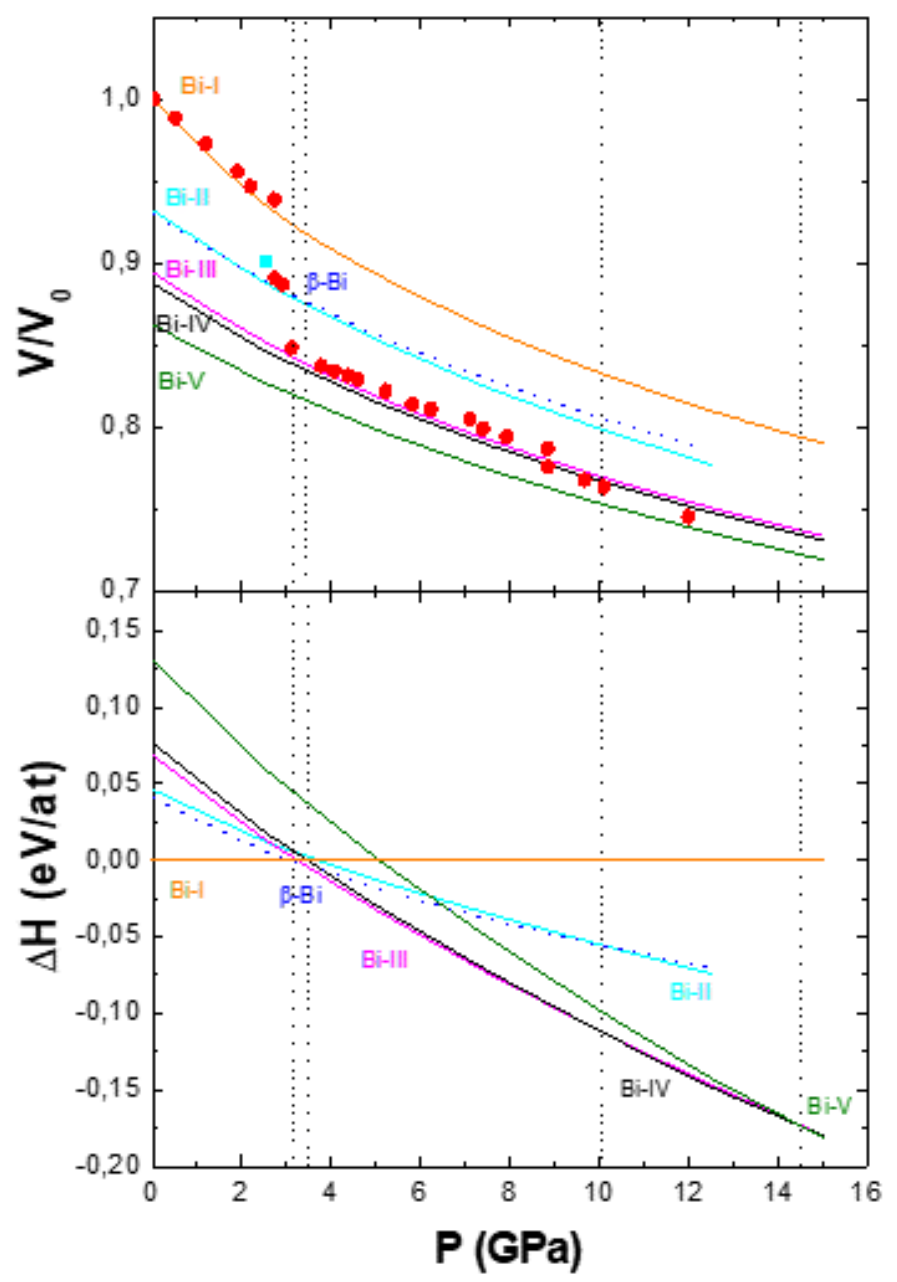

Figure 2 

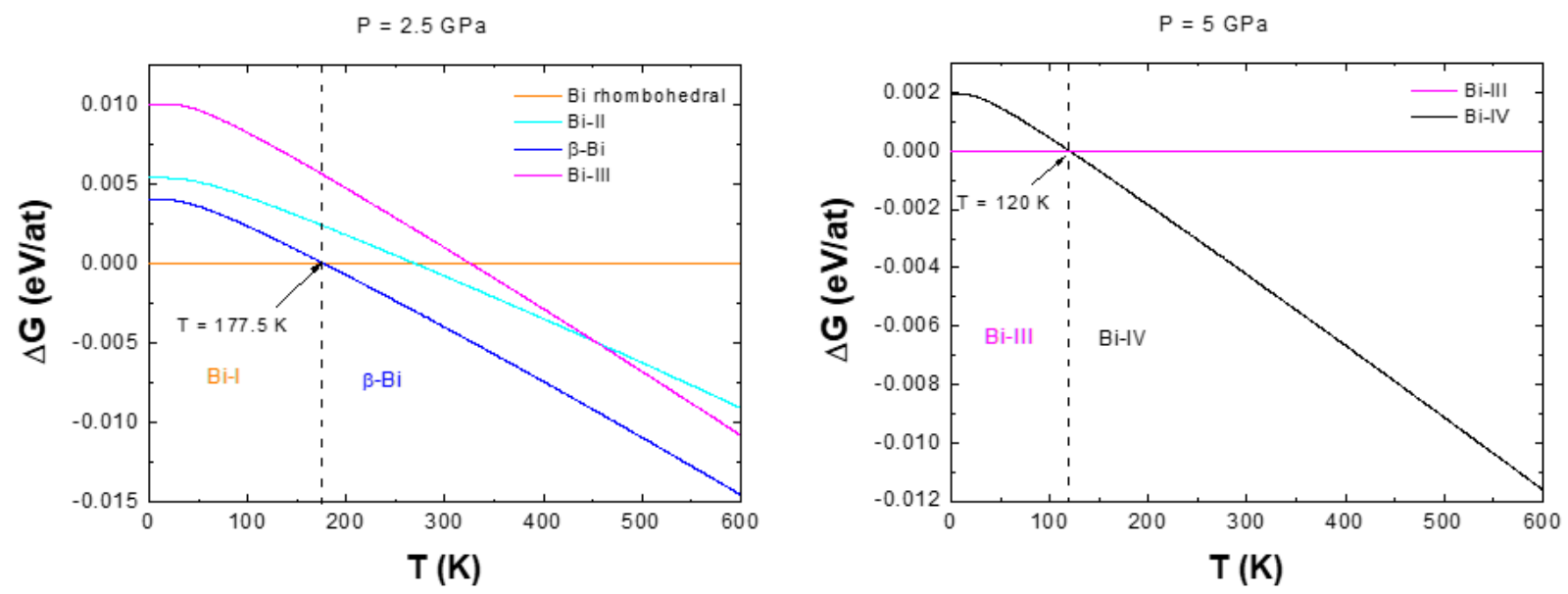

Figure 3 

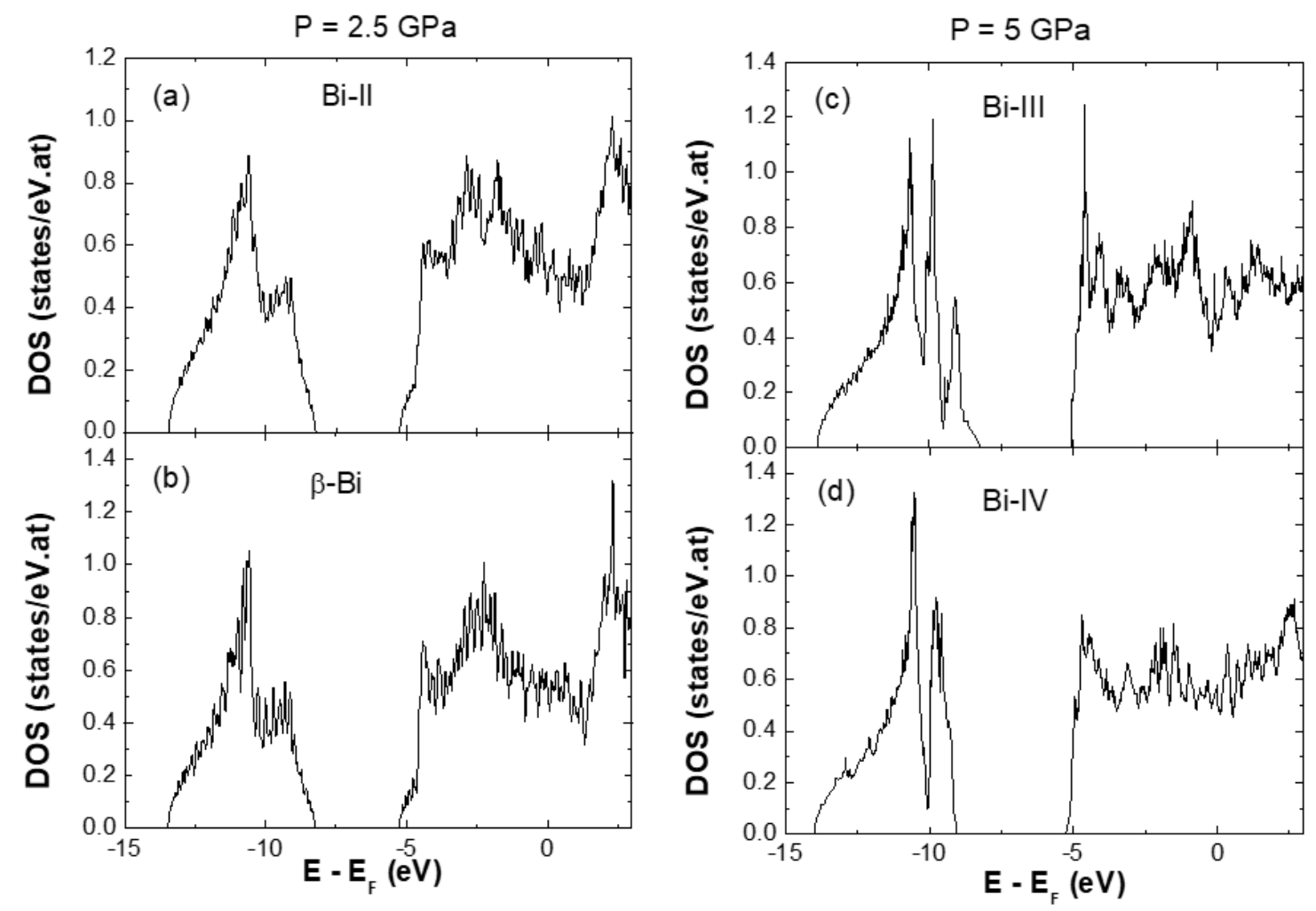

Figure 4 

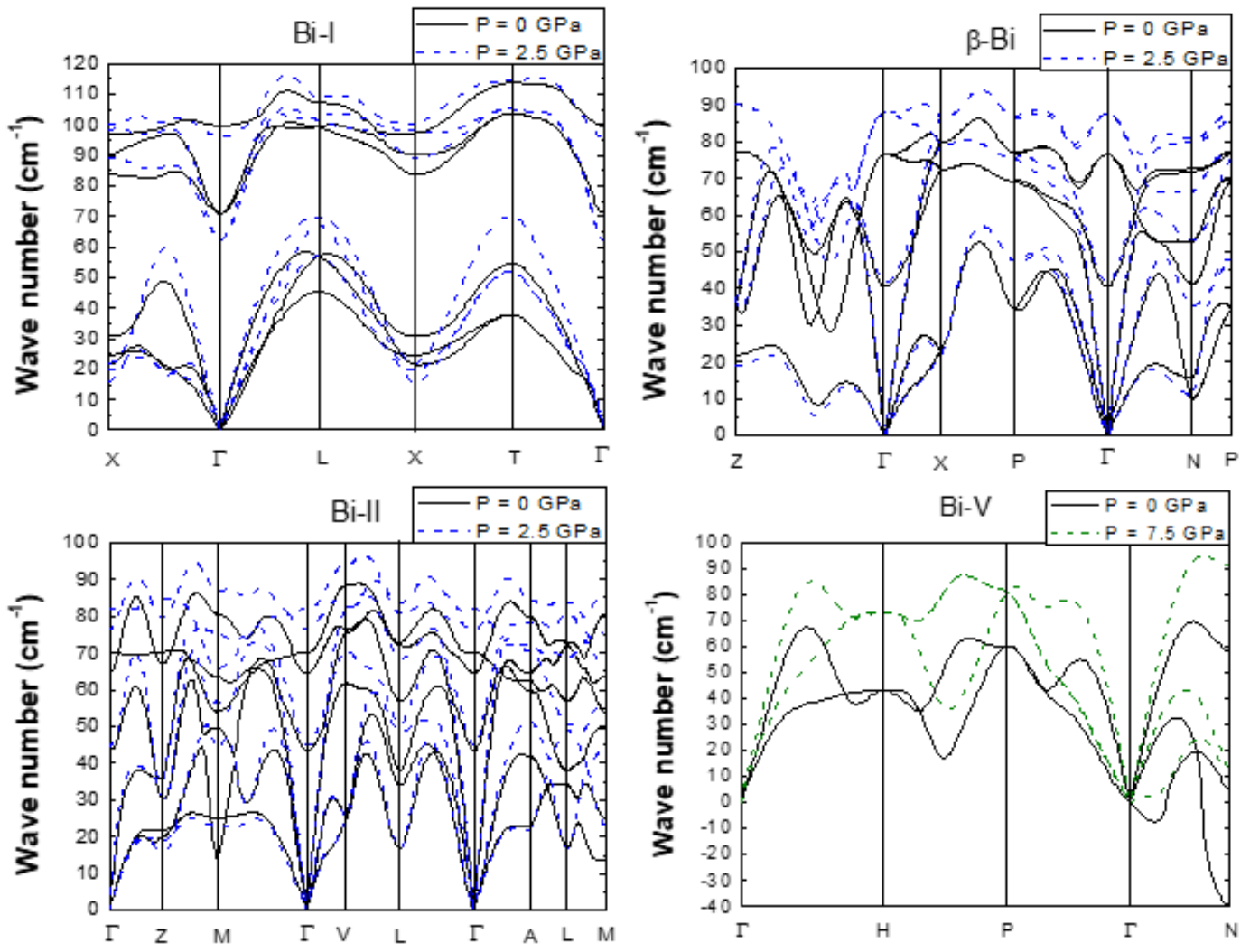

Figure 5 
Bi-III $P=0 \mathrm{GPa}$
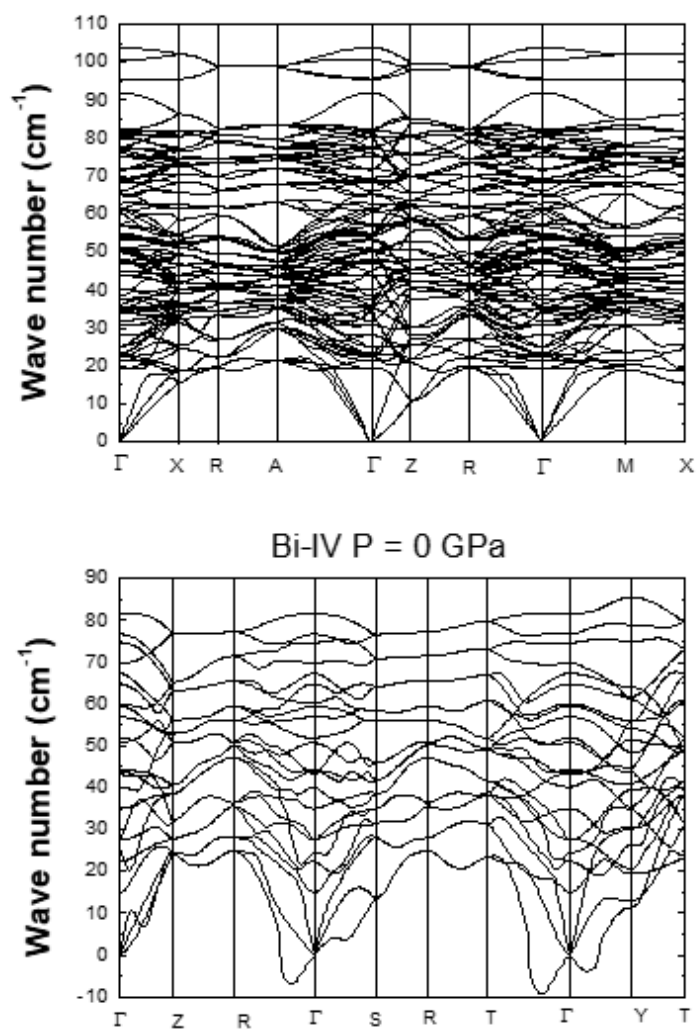

Bi-III $P=5 \mathrm{GPa}$

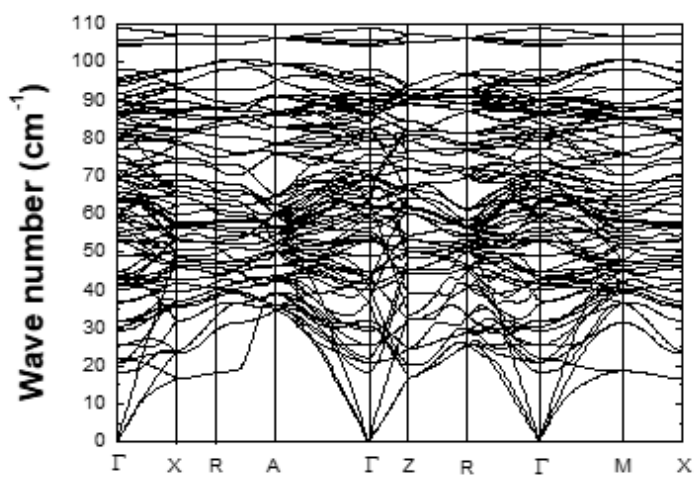

Bi-IV P $=5 \mathrm{GPa}$

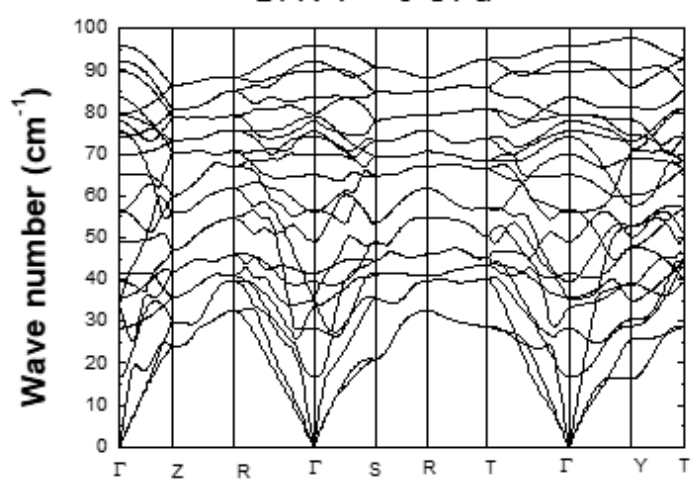

Figure 6 
Bi-I
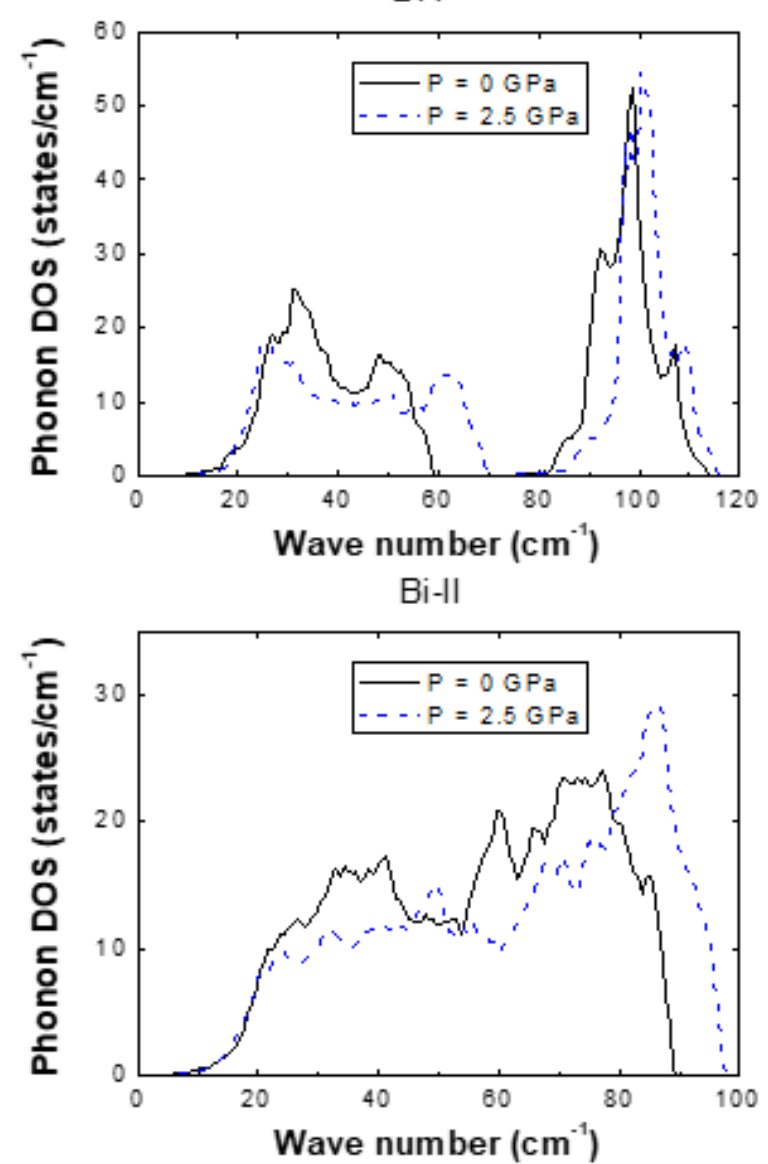

$\beta$-Bi

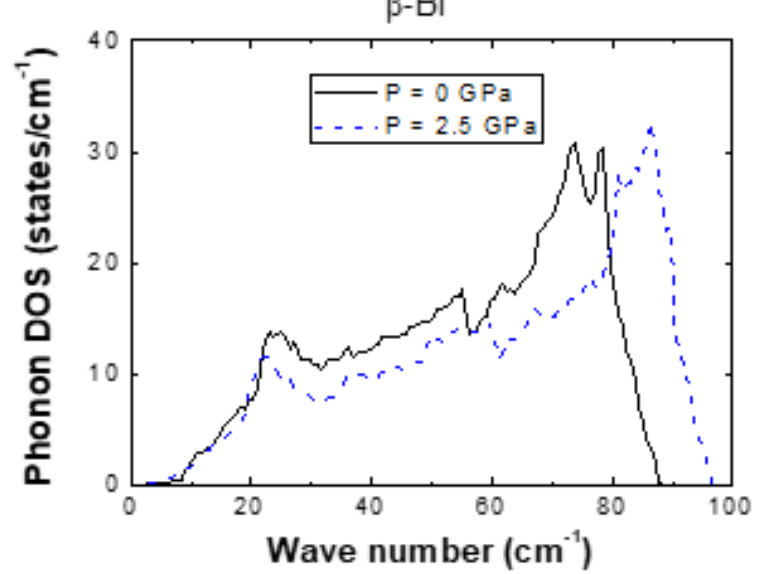

Bi-III

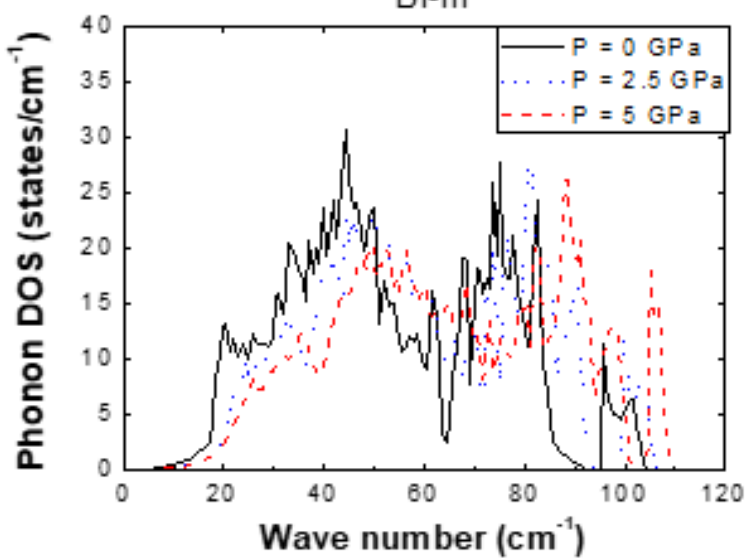

Bi-IV

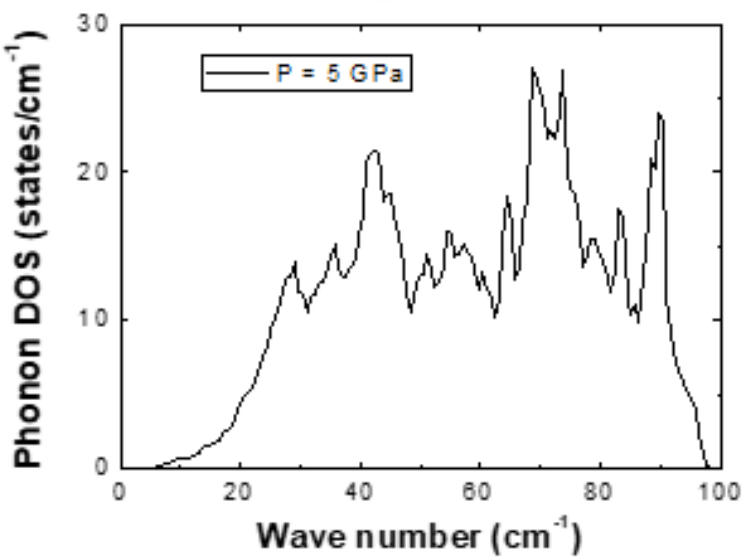

Bi-V

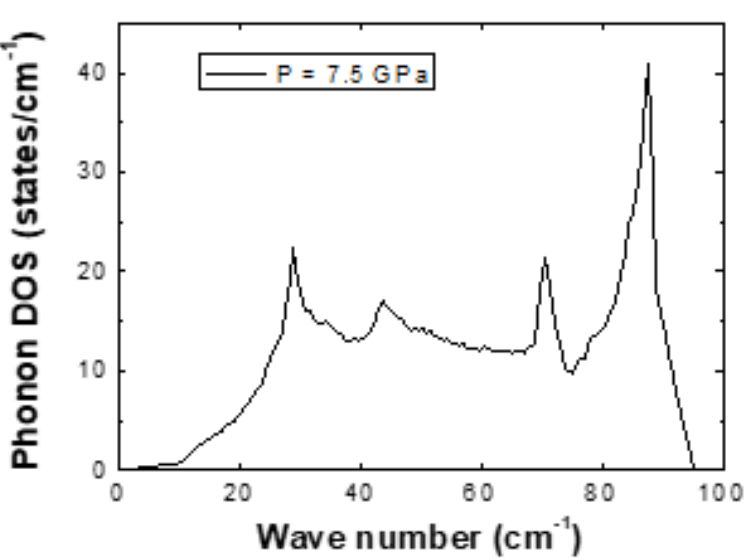

Figure 7 
Bi-III

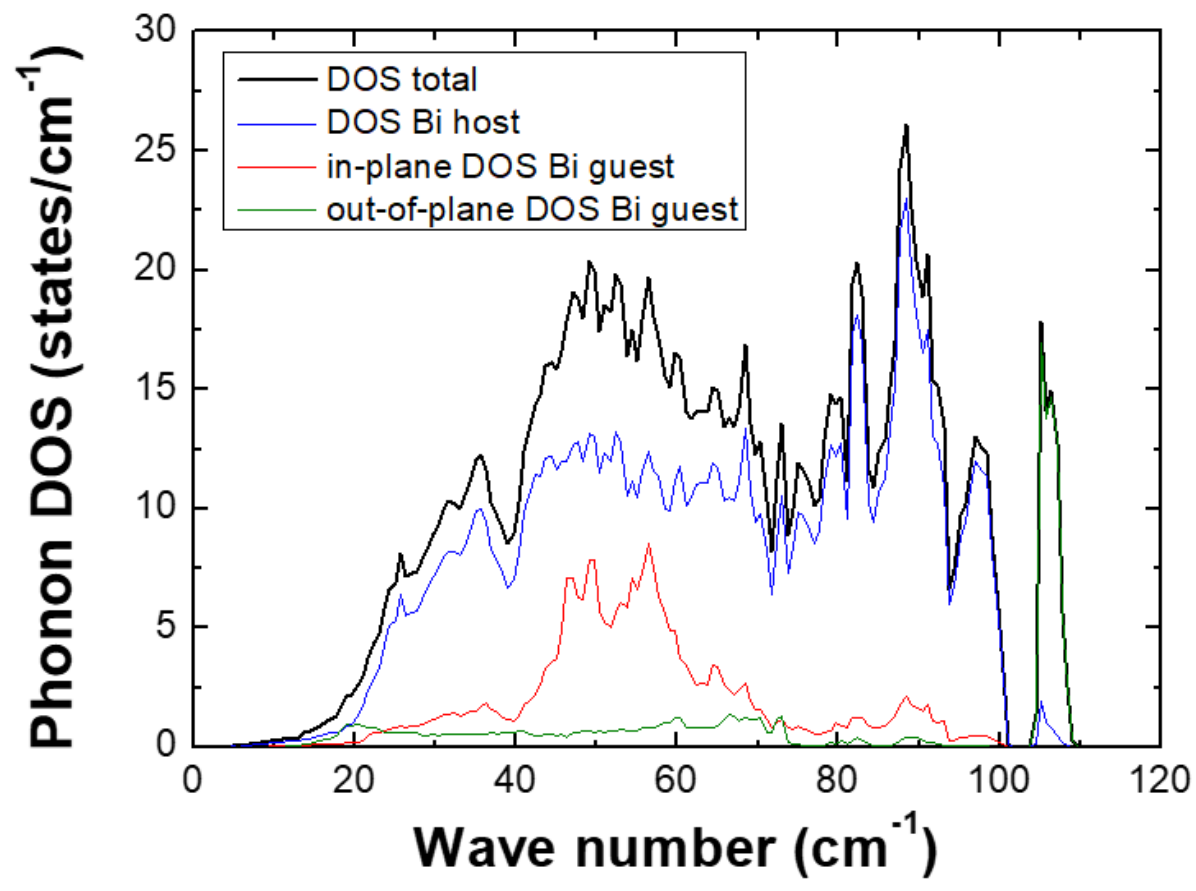

Figure 8 


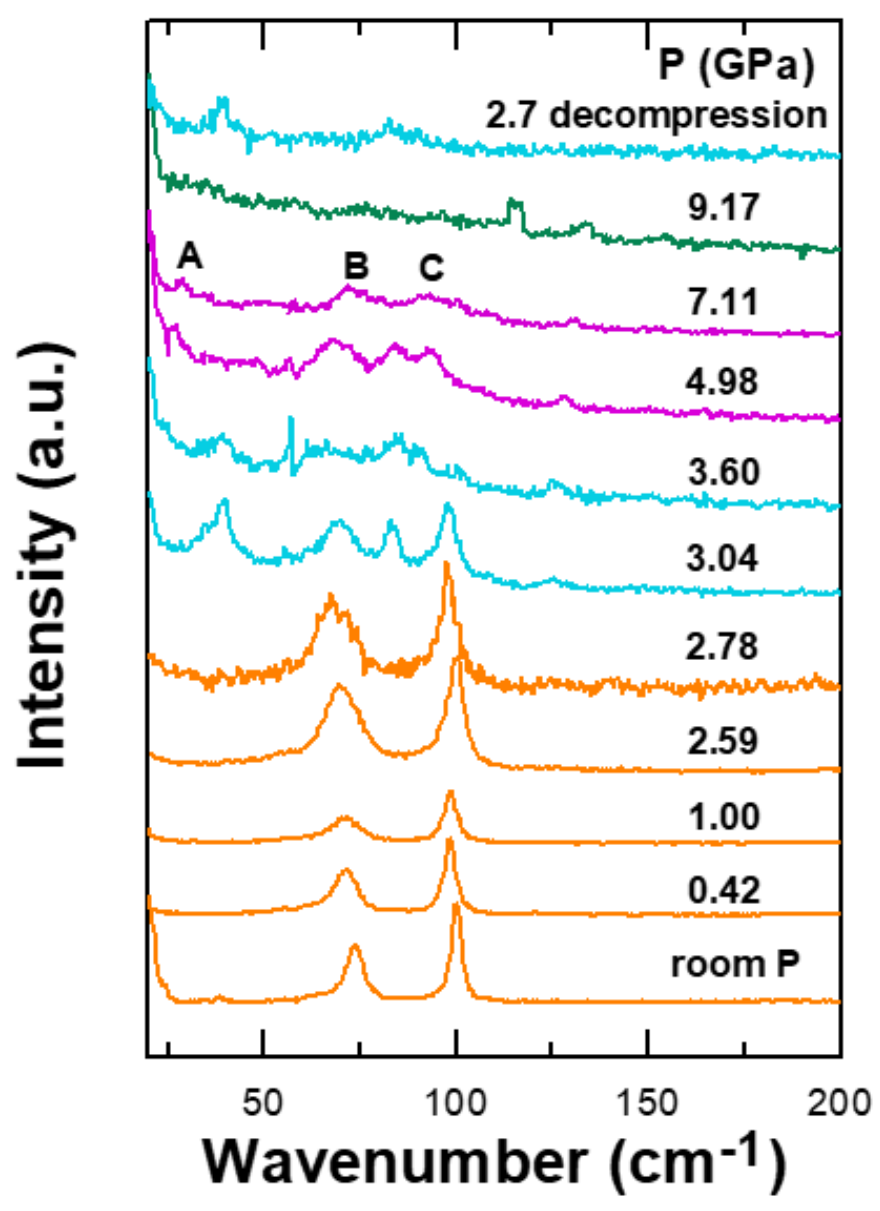

Figure 9 


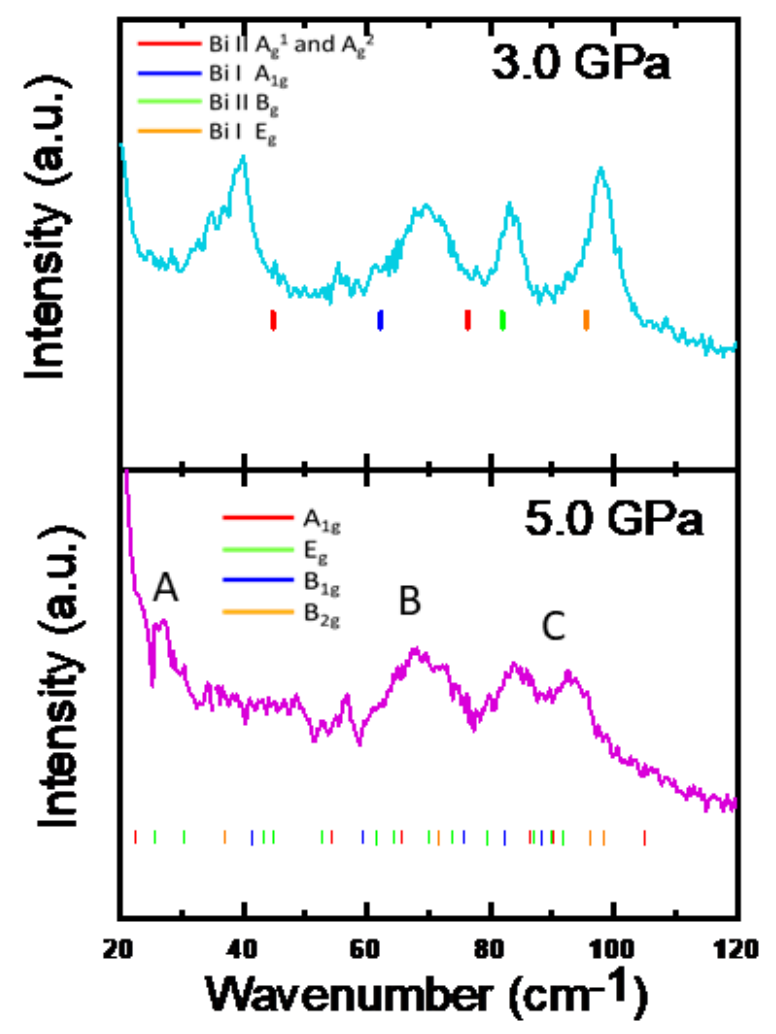

Figure 10 

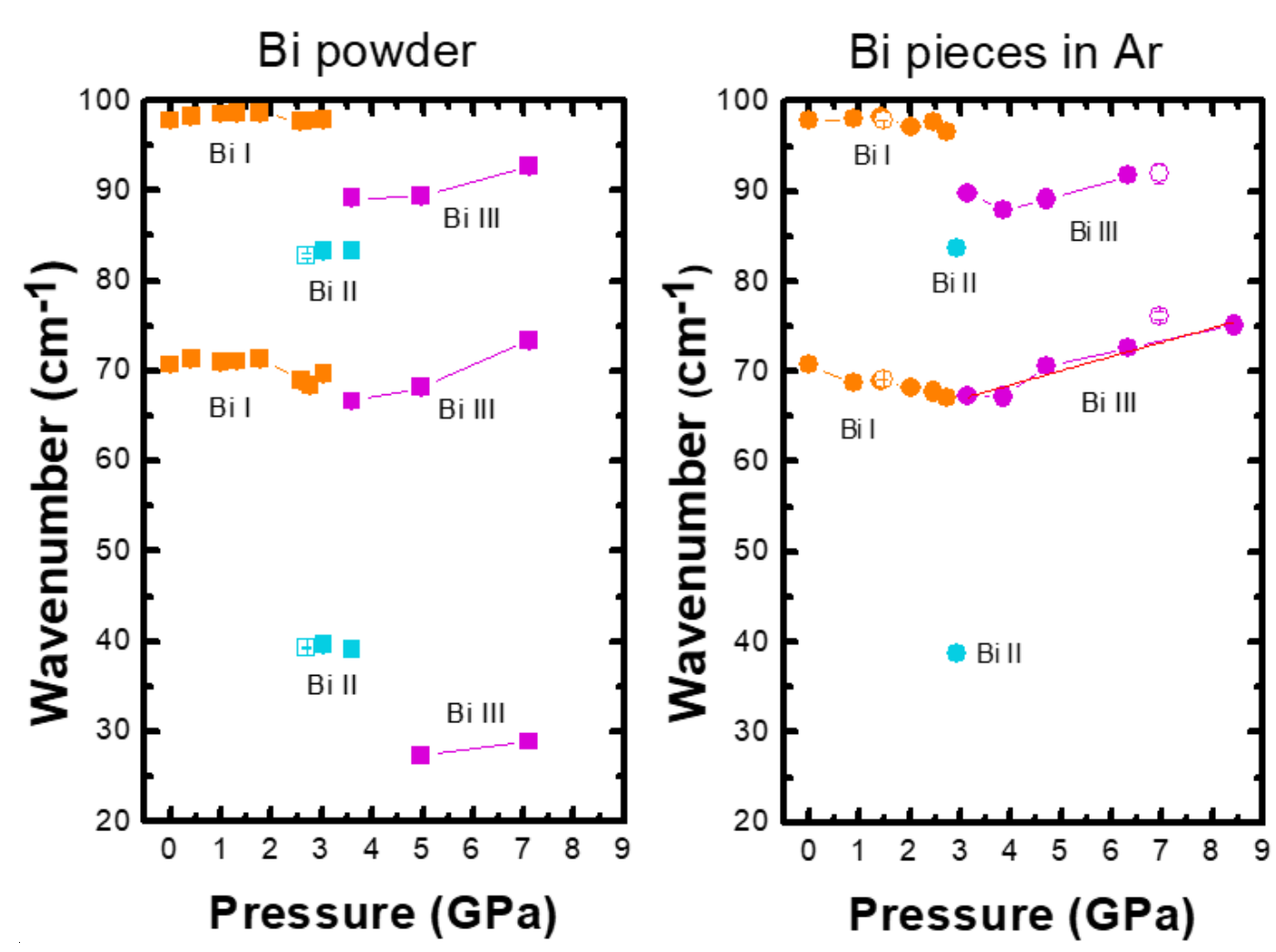

Figure 11 


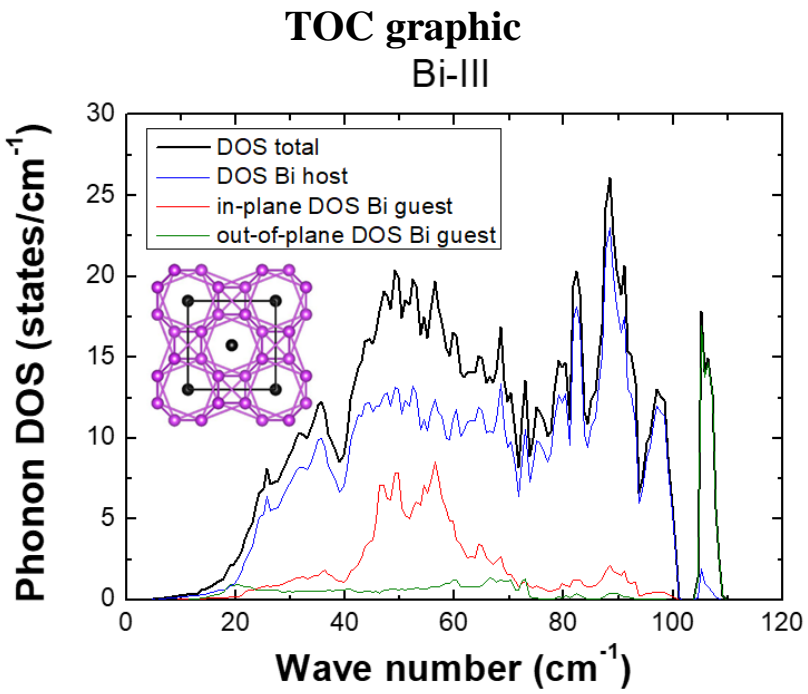

